\title{
Laminar Selectivity of the Cholinergic Suppression of Synaptic Transmission in Rat Hippocampal Region CA1: Computational Modeling and Brain Slice Physiology
}

\author{
Michael E. Hasselmo and Eric Schnell \\ Department of Psychology and Program in Neuroscience, Harvard University, Cambridge, Massachusetts 02138
}

\begin{abstract}
ACh may set the dynamics of cortical function to those appropriate for learning new information. In models of the putative associative memory function of piriform cortex, selective suppression of intrinsic but not afferent fiber synaptic transmission by $\mathrm{ACh}$ prevents recall of previous input from interfering with the learning of new input (Hasselmo, 1993). Selective cholinergic suppression may play a similar role in the hippocampal formation, where Schaffer collateral synapses in stratum radiatum (s. rad) may store associations between activity in region $\mathrm{CA} 3$ and the entorhinal cortex input to region CA1 terminating in stratum lacunosum-moleculare (s. I-m). A computational model of region CA1 predicts that for effective associative memory function of the Schaffer collaterals, cholinergic suppression of synaptic transmission should be stronger in s. rad than in s. I-m.

In the hippocampal slice preparation, we tested the effect of the cholinergic agonist carbachol $(0.01-500 \mu \mathrm{M})$ on synaptic transmission in s. rad and S. I-m. Stimulating and recording electrodes were simultaneously placed in both layers, allowing analysis of the effect of carbachol on synaptic potentials in both layers during the same perfusion in each slice. Carbachol produced a significantly stronger suppression of stimulus-evoked EPSPs in s. rad than in s. I-m at all concentrations greater than $1 \mu \mathrm{M}$. At $100 \mu \mathrm{M}$, EPSP initial slopes were suppressed by $89.1 \pm 3.0 \%$ in $\mathrm{s}$. rad, but only by $40.1 \pm 4.1 \%$ in $\mathrm{s}$. I-m. The muscarinic antagonist atropine $(1 \mu \mathrm{M})$ blocked cholinergic suppression in both layers. These data support the hypothesis that synaptic modification of the Schaffer collaterals may store associations between activity in region $\mathrm{CA} 3$ and the afferent input to region $\mathrm{CA} 1$ from the entorhinal cortex. In simulations, feedback regulation of cholinergic modulation based on activity in region CA1 sets the appropriate dynamics of learning for novel associations, and recall for familiar associations.
\end{abstract}

[Key words: ACh, hippocampus, associative memory, selforganization, synaptic modification, presynaptic inhibition, muscarinic, stratum radiatum, stratum lacunosum-moleculare]

\footnotetext{
Received Sept. 10, 1993; revised Dec. 13, 1993; accepted Dec 31, 1993

This work was supported by an Office of Naval Research Young Investigator Award and the French Foundation for Alzheimer Research. We thank Edi Barkai for experimental assistance, John Holena for simulation programming, and Matt Wilson for the data collection and graphics software.

Correspondence should be addressed to Prof. Michael E. Hasselmo, Department of Psychology, Room 950, Harvard University, 33 Kirkland Street, Cambridge, MA 02138 .

Copyright $(1994$ Society for Neuroscience $0270-6474 / 94 / 143898-17 \$ 05.00 / 0$
}

ACh suppresses excitatory synaptic transmission in many regions of the cortex, including the piriform cortex (Williams and Constanti, 1988; Hasselmo and Bower, 1992), the dentate gyrus (Yamamoto and Kawai, 1967; Kahle and Cotman, 1989; Burgard and Sarvey, 1990), region CA1 of the hippocampus (Hounsgaard, 1978; Valentino and Dingledine, 1981; Dutar and Nicoll, 1988; Blitzer et al., 1990; Sheridan and Sutor, 1990), and neocortical structures (Brocher et al., 1992). The memory impairment caused by muscarinic antagonists or lesions of cortical cholinergic innervation (Beatty and Carbone, 1980; Walker and Olton, 1984; Spencer et al., 1985; Kopelman, 1986; Hagan and Morris, 1989) may be due to blockade of this cholinergic suppression. Computational modeling suggests that selective cholinergic suppression of synaptic transmission may be essential to associative memory function within cortical structures (Hasselmo et al., 1992; Hasselmo, 1993, 1994; Hassclmo and Bower, 1993; Barkai and Hasselmo, 1994b).

Associative memory function involves the learned capacity for a specific pattern of activity in one population of cortical neurons to elicit an associated pattern of activity in another population of cortical neurons (Grossberg, 1970; Anderson, 1972, 1983; Amit, 1988; Kohonen, 1988; Hasselmo et al., 1992; Hasselmo, 1993, 1994). Learning of these associations is commonly obtained by strengthening excitatory synapses between neurons based on concurrent pre- and postsynaptic activity (e.g., the Hebb rule). However, to prevent recall of previously learned associations from altering the new association, the modifiable synapses must not be the predominant influence on postsynaptic activity during learning (Hasselmo et al., 1992; Hasselmo, 1993). If modifiable synapses are the predominant influence on postsynaptic activity during learning, then these synapses undergo self-organization (Von der Malsburg, 1973; Grossberg, 1976; Linsker, 1988; Miller et al., 1989; Hasselmo, 1994).

In the piriform (olfactory) cortex (see Fig. 1), ACh selectively suppresses synaptic transmission at excitatory intrinsic synapses between pyramidal cells, while having little effect on synaptic transmission at afferent fibers arising from the olfactory bulb (Hasseimo and Bower, 1992). In models of piriform cortex (Hasselmo et al., 1992; Barkai and Hasselmo, 1994a,b; Hasselmo, 1993, 1994), suppression of intrinsic fiber synaptic transmission during learning prevents the recall of previously stored associations from interfering with the learning of new associations. At the same time, the weaker influence on afferent fiber synapses allows them to undergo self-organization, and to set the pattern of activity to be learned (Hasseimo, 1994).

These results may apply to cholinergic modulation of memory function in the hippocampus as well. As shown in Figure 1, the 
pyramidal cells of region CAl receive excitatory synaptic input from different cortical regions in different layers. In the proximal layer, stratum radiatum (s. rad), these neurons receive excitatory synapses of the Schaffer collaterals, which arise from region CA3 (see Amaral and Witter, 1989, for review). In the distal layer, stratum lacunosum-moleculare (s. l-m), these neurons receive excitatory synapses of the perforant pathway arising from layer III of the entorhinal cortex (Lorente de No, 1938; Steward, 1976; Witter et al., 1988). A considerable amount of research has focused on the possible Hebbian nature of synaptic modification at the Schaffer collaterals (Kelso et al., 1986; Wigstrom et al., 1986; Gustafsson and Wigstrom, 1988). Some researchers have proposed that this Hebbian synaptic modification underlies heteroassociative memory function at the Schaffer collaterals, allowing these synapses to store associations between the activity of region CA3 and the afferent input from entorhinal cortex (Levy, 1989; Eichenbaum and Buckingham, 1990; Levy et al., 1990; McNaughton, 1991). This could allow the region to perform a comparison of the output of region CA3 with the current afferent input from the entorhinal cortex (Levy, 1989; Eichenbaum and Buckingham, 1990; Levy et al., 1990). In contrast, other models have proposed that the Schaffer collaterals undergo competitive self-organization (Rolls, 1991).

The possible comparison function of region $\mathrm{C} \Lambda 1$ could allow activity in this area to reflect the novelty or familiarity of a pattern of afferent input, and thereby to regulate its own cholinergic modulation for the appropriate dynamics of learning or recall. This comparison function requires that the Schaffer collaterals have the capacity to perform effective heteroassociative memory function, rather than only to undergo self-organization. The simulations presented here show that if the Schaffer collaterals have heteroassociative memory function, this would require that $\mathrm{ACh}$ should suppress synaptic transmission more strongly in s. rad than in s. $1-\mathrm{m}$. Previous studies have demonstrated a strong cholinergic suppression of synaptic transmission at the Schaffer collateral-CA1 synapse (Hounsgaard, 1978; Valentino and Dingledine, 1981; Dutar and Nicoll, 1988; Sheridan and Sutor, 1990), but no studies have analyzed the effects of cholinergic modulation in s. $1-\mathrm{m}$. In the experimental work presented here, we demonstrate that cholinergic suppression of synaptic transmission is indeed stronger in stratum radiatum than in stratum lacunosum-moleculare, supporting the theory of associative memory function at the Schaffer collaterals.

\section{Materials and Methods}

\section{Computational modeling}

The effect of the cholinergic suppression of synaptic transmission in stratum radiatum (s. rad) and stratum lacunosum-moleculare (s. 1-m) on the function of region CA1 was explored in a simple computational simulation. The simulation contained representations of entorhinal cortex layer III and regions CA1 and CA3 of the hippocampus, with excitatory and inhibitory synaptic connectivity and the effects of cholinergic modulation. As shown in Figure 2, the simulation incorporated both the perforant pathway input from entorhinal cortex, terminating in stratum lacunosum-moleculare of region $\mathrm{CA} 1$, as well as the Schaffer collateral input from region $\mathrm{CA} 3$, terminating in stratum radiatum of region CA1. These inputs influenced the activity of pyramidal cells in region CA1.

Simulations evaluated how the cholinergic suppression of synaptic transmission influenced the self-regulated heteroassociative memory function of Schaffer collateral synapses. Thus, in these simulations, activity patterns were induced sequentially in the entorhinal cortex and region CA3. When the Schaffer collaterals showed effective heteroassociative memory function, they could store associations between the activity in region $\mathrm{CA} 3$ and the coincident activity induced in region

\section{Hippocampus}

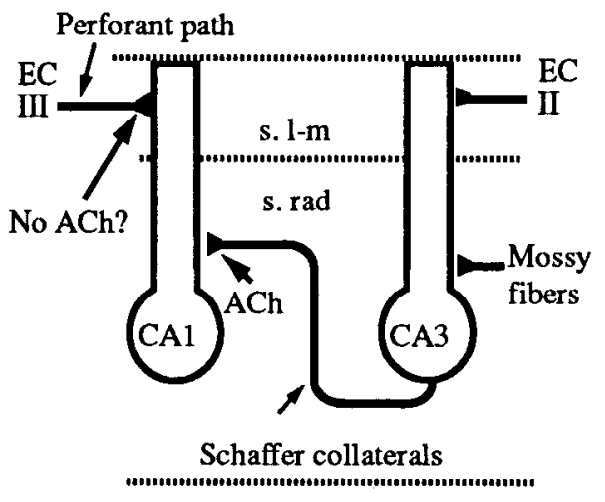

\section{Piriform cortex}

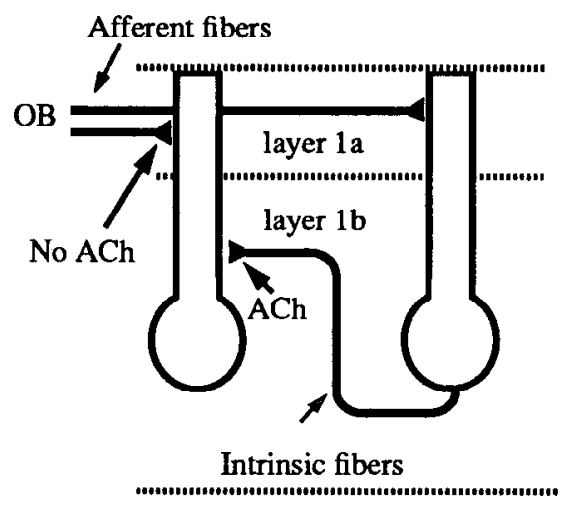

Figure 1. A, Schematic representation of hippocampal region CA1 showing the laminar segregation of excitatory synapses. Perforant path fibers from the entorhinal cortex layer III $(E C I I I)$ terminate on the distal dendrites of pyramidal cells in stratum lacunosum-moleculare $(s$. $l-m)$. Schaffer collateral axons arising from region CA3 pyramidal cells terminate on the proximal dendrites of pyramidal cells in stratum radiatum $(s . \mathrm{rad})$. In simulations, selective cholinergic suppression of Schaffer collaterals allows them to store associations between activity in region $\mathrm{CA} 3$ and the input from entorhinal cortex. Cholinergic suppression of synaptic transmission at the Schaffer collaterals has been demonstrated $(A C h)$, but cholinergic suppression of synaptic transmission at the perforant path synapses in $\mathrm{s}$. 1-m has not been studied previously (NoACh?). B, Schematic representation of the piriform cortex showing laminar segregation of excitatory synapses. Afferent fibers from the olfactory bulb $(O B)$ terminate in layer $1 \mathrm{a}$, while intrinsic and association fibers arising from cortical pyramidal cells terminate in layer 1b. Cholinergic suppression of synaptic transmission in layer $1 \mathrm{~b}(A C h)$ but not layer $1 \mathrm{a}(\mathrm{No} A C h$ ) has been demonstrated previously (Hasselmo and Bower, 1992, 1993).

CA1 by input from the entorhinal cortex. The recall of these associations could be tested by inducing activity in region CA3 alone and evaluating how closely the activity spreading into region CA1 resembled the activity previously provided by input from the entorhinal cortex.

Most previous models of autoassociative and heteroassociative memory function externally set the dynamics appropriate for learning or recall within the network (Anderson, 1972; Hopfield, 1984; Amit, 1988; Kohonen, 1988). In the simulations presented here, however, the dynamics of learning and recall were determined entirely by internal selfregulation of cholinergic modulation. Thus, when the sequence of patterns was presented, the network could respond with appropriate dynamics for learning or recall. When the pattern pair was novel, cholinergic modulation remained high, allowing appropriate dynamics for learning. When the pattern pair was familiar, the output of the network decreased cholinergic modulation, thereby setting appropriate dynamics for recall. Thus, the testing of heteroassociative memory function did 


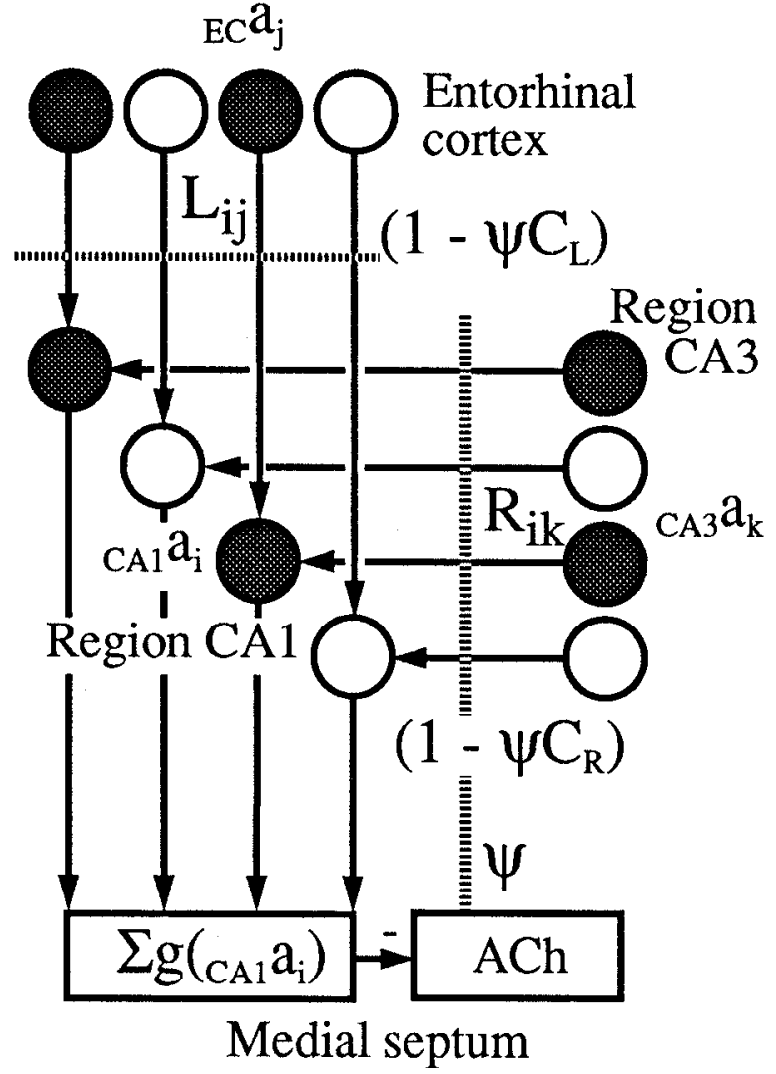

Figure 2. Schematic representation of the simulation of region CAl. The membrane potential of CAl pyramidal cells $\mathrm{CA}_{\mathrm{A}} a_{i}$ is influenced by excitatory input from entorhinal cortex $(E C)$ via synapses of the perforant path in $\mathrm{s}$. I-m $\left(L_{i j}\right)$ and from region CA3 via synapses of the Schaffer collatcrals in s. rad $\left(R_{i k}\right)$. In addition, these neurons are influenced by feedforward inhibition activated by entorhinal input and by CA3 input as well as feedback inhibition triggered by the output of other CA1 neurons (see Materials and Methods). Cholinergic suppression of synaptic transmission due to $\mathrm{ACh}$ released from neurons of the medial septum and vertical limb of the diagonal band of Broca is represented by $\left(1-\psi C_{L}\right)$ in s. $1-\mathrm{m}\left(L_{i j}\right)$ and by $\left(1-\psi C_{R}\right)$ in $\mathrm{s}$. $\operatorname{rad}\left(R_{i k}\right)$. The summed output of region CA1 $\left.\left[\Sigma g_{\left(C_{1}\right.} a_{i}\right)\right]$ decreases the level of cholinergic modulation from the medial septum. This allows feedback regulation of cholinergic modulation by region CA1 to determine directly when it is in a state of learning and when it is in a state of recall (see Materials and Methods).

not involve a separate stage of testing recall, but could be performed by presenting only the $\mathrm{CA} 3$ component of previously learned pattern pairs.

\section{Spread of activity}

In the model, an activation variable $(a)$ represented the depolarization of the membrane potential of each pyramidal cell from resting potential. This variable was influenced by the sum of excitatory and inhibitory synaptic inputs,

$$
\begin{aligned}
\mathrm{CA}_{1} a_{i}(t+1)= & \sum_{j=1}^{n}\left(L_{i j}-{ }_{\mathrm{EC}} H_{i j}\right) * g\left(_{\mathrm{EC}} a_{j}(t)\right) \\
+ & \sum_{k=1}^{n}\left(R_{i k}-{ }_{\mathrm{CA}_{3}} H_{i k}\right) * g\left({ }_{C A 3} a_{k}(t)\right) \\
& \sum_{t=1}^{n}{ }_{\mathrm{CA} 1} H_{i l} * g\left(_{\mathrm{CA} 1} a_{j}(t)\right)
\end{aligned}
$$

where ${ }_{\mathrm{CA} 1} a_{i}$ represents the activity of neuron $i$ in region CA1, ${ }_{\mathrm{F}} a_{j}$ represents the activity of neuron $j$ in entorhinal cortex, and ${ }_{\mathrm{CA} 3} a_{k}$ represents the activity of neuron $k$ in region $\mathrm{CA} 3$, as shown in Figure 2 . The input/ outpul function $g($.$) was a nonlinear threshold function with the follow-$ ing characteristics: for ${ }_{\mathrm{EC}} a_{j}-\theta>0, g\left(\mathrm{EC} a_{j}\right)={ }_{\mathrm{EC}} a_{j}-\theta$; for ${ }_{\mathrm{EC}} a_{j}-\theta \leq$ $0, g\left({ }_{\mathrm{EC}} a_{j}\right)=0$. The threshold $\theta$ was set at 0.4 for region $\mathrm{CA} 1 . L_{i j}$ represents the strength of each synapse in stratum lacunosum-moleculare from neuron $j$ in entorhinal cortex to neuron $i$ in region CA1, as shown in Figure 2. $R_{i k}$ represents the strength of each synapse in stratum radiatum from neuron $k$ in region $\mathrm{CA} 3$ to neuron $i$ in region $\mathrm{CA} 1$.

For clarity in the examples showing heteroassociative memory function, the synaptic connectivity matrix in s. $1-\mathrm{m}\left(L_{i j}\right)$ was assumed to consist of the identity matrix, such that the activity in entorhinal cortex spread to the CA1 pyramidal cells without a change in pattern. In additional simulations, the $L_{i j}$ matrix started with random initial connectivity, allowing more complex transformations of entorhinal cortex output to be formed by self-organization of the synapses in $\mathrm{s}$. $1-\mathrm{m}$. In keeping with physiological evidence showing that synapses in $\mathrm{s}$. 1-m have a much weaker influence than synapses in s. rad (Andersen et al., 1966; Doller and Weight, 1982; Yeckel and Berger, 1990; Colbert and Levy, 1992), each synapse in s. l-m had a total strength of 0.4 in simulations of pure heteroassociative memory function. Since $L_{i j}$ was the identity matrix in these simulations, the total input to each CAl neuron from $\mathrm{s}$. $1-\mathrm{m}$ was therefore 0.4 . In contrast, the synaptic connectivity matrix in s. rad $\left(R_{i k}\right)$ started with random initial connection strengths with a mean of 0.157 , giving an average initial input of $6 * 0.157=0.942$ for patterns in which six neurons in region $\mathrm{CA} 3$ had output 1.0. Synapses of $R_{i k}$ could not be weaker than 0.1 or stronger than 0.5 . This difference in strength of synaptic input partly results from the grcater distance from the cell body of synapses in $\mathrm{s}$. l-m versus synapses in $\mathrm{s}$. rad. However, analysis of the exact role of the laminar segregation of these synaptic influences will require more detailed biophysical simulations.

In Equation 1, ${ }_{\mathrm{EC}} H_{i j}$ represents feedforward inhibition mediated by interneurons of $\mathrm{s}$. 1-m (Lacaille et al., 1988) activated by perforant path synapses, ${ }_{C_{A} 3} H_{i k}$ represents feedforward inhibition in region $\mathrm{CAl}$ due to activity of the Schaffer collaterals from region $\mathrm{CA} 3$, and ${ }_{\mathrm{CAl}} H_{i l}$ represents feedback inhibition within region CAl. Each inhibitory connectivity matrix was uniform, with individual components of strength 0.1 . In some cases, inhibition could induce oscillatory activity within the simulation. In more complex simulations of cortical structures, inhibitory influences with different time constants can induce oscillations with peaks in the power spectra resembling those observed in EEG recordings (Traub et al., 1992; Wilson and Bower, 1992; Liljenstrom and Hasselmo, 1993). However, the simplified simulation presented here does not systematically attempt to replicate the broad range of evidence for $\theta$-type oscillations in the hippocampal formation (Bland and Colom, 1993). Accurate replication of such oscillations will require development of more detailed simulations of region CAl similar to those developed for piriform cortex (Wilson and Bower, 1992; Barkai and Hasselmo, 1994a,b; Liljenstrom and Hasselmo, 1993) and region CA3 (Traub et al., 1992).

\section{Synaptic modification}

The strengths of individual synapses were modified according to a learning rule with a Hebbian component allowing synapses to grow dependent upon coincident pre- and postsynaptic activity. Long-term potentiation with Hebbian properties has been described in s. rad of region CA1 (Kelso et al., 1986; Wigstrom et al., 1986; Gustafsson and Wigstrom, 1988). Levy and Colbert (1992) have shown that responses to Schaffer collateral stimulation can be associatively potentiated with stimulation in $\mathrm{s.} 1 \mathrm{-m}$. In the simulation, the synapses also undergo a decay proportional to both postsynaptic activity and the strength of the synapse. A learning rule of this sort has been suggested previously on the basis of experimental data (Levy et al., 1990) and for stability during learning in analytical descriptions (Grossberg, 1976). While long-term potentiation has been demonstrated in s. 1-m (Levy and Colbert, 1992), these synapses were not modified in the simulations presented here. In other simulations, modification of these synapses allowed them to undergo self-organization, thereby altering the representation of entorhinal cortex input in region $\mathrm{CA} 1$. The learning rule took the following form for synapses in $\mathrm{s} . \operatorname{rad}\left(R_{i k}\right)$ :

$$
\begin{aligned}
R_{i k}(t+1)=\eta[ & \left(C_{\mathrm{CA} 1} a_{i}(t)-\theta\right) * g\left({ }_{\mathrm{CA} 3} a_{k}(t)\right) \\
& \left.-\mu\left({ }_{\mathrm{CA} 1} a_{i}(t)-\theta\right) * R_{i k}(t)\right]+R_{i k}(t)
\end{aligned}
$$

As reflected in the notation, the modification threshold $\theta$ took the same value as the output threshold, that is, 0.4 . The gain of the learning rule, $\eta$, was set at 1.0 for simulations, while the coefficient of the decay of 
synaptic strength, $\mu$, was set at 0.04 . In simulations of pure heteroassociative memory function, synapses in $\mathrm{s}$. $1-\mathrm{m}$ were not modifiable.

\section{Cholinergic modulation}

The model contained representations of the physiological effects of acetylcholine within hippocampal region CA1, as well as a representation of the feedback regulation of the cholinergic modulation of this region. Note that these effects of cholinergic modulation are applied in a diffuse manner throughout the simulation, in keeping with the broadly distributed nature of the cholinergic innervation of the hippocampus arising from the medial septum and the vertical limb of the diagonal band of Broca (Frotscher and Leranth, 1985; Gaykema et al., 1990), and evidence in neocortical structures supporting the possibility of volume transmission (Umbriaco et al., 1992). Four different effects of acetylcholine demonstrated in experimental work were incorporated as follows.

Cholinergic suppression of synaptic transmission. Previous experimental work has demonstrated cholinergic suppression of synaptic transmission in s. rad. (Hounsgaard, 1978; Valentino and Dingledine, 1981; Dutar and Nicoll, 1988; Sheridan and Sutor, 1990), but the effects of cholinergic modulation on synaptic transmission in $\mathrm{s}$. $1-\mathrm{m}$ have been explored only in the experiments presented here. In the simulation, the amount of cholinergic suppression of synaptic transmission was represented by the constants $C_{L}$ for maximal suppression in s. 1-m and $C_{R}$ for maximal suppression in s. rad, as shown in Figure 2. The feedback regulation of cholinergic modulation described below could cause suppression to go to values lower than these maximal values during the simulation. Thus, the maximal suppression $\left(C_{L}, C_{R}\right)$ means the percentage decrease in synaptic transmission in either layer during maximum cholinergic modulation ( $\psi=1$ as described in Eq. 3 below). The maximal suppression parameters were varied extensively between 0 and 1 in simulations (e.g., see Figs. 4-7) to explore the effect of different amounts of cholinergic suppression of synaptic transmission.

Cholinergic suppression of neuronal adaptation. Experimental work has demonstrated that cholinergic modulation increases the excitability of neurons in region CAl due to suppression of voltage- and calciumdependent potassium currents underlying adaptation (Benardo and Prince, 1982; Madison and Nicoll, 1984; Madison et al., 1987). Characterization of effects of this type in the piriform cortex (Barkai and Hasselmo, 1994a) shows that cholinergic modulation does not appear to change the threshold of the initial response of neurons to excitatory stimulation, but changes the threshold of the adapted response of ncurons. This is approximated in the present simulations by decreasing the output threshold $\theta$ proportional to the amount of cholinergic modulation (with a maximum decrease of $C_{\theta}=0.64$ in all simulations shown here).

Cholinergic enhancement of synaptic modification. Experimental work has demonstrated an enhancement of synaptic modification in hippocampal region CA1 in the presence of cholinergic modulation (Burgard and Sarvey, 1989; Blitzer et al., 1990; Huerta and Lisman, 1993), possibly due to influences of cholinergic modulation on NMDA currents (Markram and Segal, 1991). This enhancement of synaptic modification by cholinergic modulation has been incorporated as a decrease in the threshold of synaptic modification (with a maximum decrease of $C_{\theta}=$ 0.64 in all simulations shown here), and an increase in the coefficient of learning (as described in Eq. 5).

Cholinergic suppression of inhibitory synaptic transmission. Expcrimental evidence suggests that cholinergic modulation simultaneously activates inhibitory interneurons and decreases inhibitory synaptic transmission (Pitler and Alger, 1992). In an attempt to represent the net effect of this modulation, the level of feedforward and feedback inhibition within the model was suppressed proportional to the level of cholinergic modulation, with a maximum decrease of $C_{H}=0.8$ for all types of inhibition in all simulations shown here. While the values for cholinergic suppression of excitatory synaptic transmission were varied extensively, the other parameters for maximal effects of cholinergic modulation were kept at the same value in these simulations.

Feedback regulation of cholinergic modulation. As a first approximation of the feedback regulation of the activity of cholinergic neurons in the medial septum and vertical limb of the diagonal band of Broca, the level of cholinergic modulation was decreased proportional to summed activity in region CA1. Activity in the medial septum has been shown to be inhibited by stimulation of the fimbria, which presumably activates efferent fibers from the hippocampus (McLennan and Miller, $1974 \mathrm{a}, \mathrm{b})$. In these simulations, the amount of activity of cholinergic neurons in the medial septum and diagonal band was computed in the following form:

$$
\psi=\left[1+\exp \left(\xi\left(\sum_{i=1}^{n} g\left(\mathrm{CAl}_{\mathrm{I}} a_{i}\right)-\nu\right)\right)\right]^{-1},
$$

where $\psi$ represents the level of acetylcholine released within hippocampal region CAl. This equation shows that as the summed output of pyramidal neurons in region CAI increases, the level of cholinergic modulation decreases. The equation approaches maximum value when there is little or no CAl output, decreases to half strength when the summed output is equal to the threshold $v$ (set here at 3.0), and approaches 0 as the summed output becomes large. The slope of this function is determined by the gain term $\xi$ (set here at 2.0 ).

In summary, with cholinergic modulation included, the output function of individual neurons took the following form: for ${ }_{\mathrm{EC}} a_{j}-(1-$ $\left.\psi C_{\theta}\right) * \theta>0, g\left({ }_{\mathrm{EC}} a_{j}\right)={ }_{\mathrm{EC}} a_{j}-\left(1-\psi C_{\theta}\right) * \theta$; for ${ }_{\mathrm{EC}} a_{j}-\left(1-\theta C_{\theta}\right) * \theta \leq 0$, $g\left({ }_{\mathrm{EC}} a_{j}\right)=0$.

With cholinergic modulation, the activation equation took the form

$$
\begin{aligned}
\mathrm{CA} a_{i}(t+1)= & \sum_{j=1}^{n}\left[\left(1-\psi C_{L}\right) L_{i j}-\left(1-\psi C_{H}\right) H_{i j}\right] * g\left(_{\mathrm{EC}} a_{j}(t)\right) \\
& +\sum_{k=1}^{n}\left[\left(1-\psi C_{R}\right) R_{i k}-\left(1-\psi C_{H}\right) H_{i k}\right] * g\left({ }_{\mathrm{CA} 3} a_{k}(t)\right) \\
& -\sum_{i=1}^{n}\left(1-\psi C_{H}\right) H_{i t} * g\left({ }_{\mathrm{CA}} a_{i}(t)\right)
\end{aligned}
$$

For example, when there was no region CAl output, feedback regulation allowed acetylcholine to approach a maximum level $(\psi \approx 1)$. In this case, when maximal suppression in s. rad was $0.8\left(C_{R}=0.8\right)$, then synaptic transmission at the synapses $R_{i k}$ would decrease to just over 0.2 of their normal value $\left(1-\psi C_{L} \approx 0.2\right)$.

With cholinergic modulation, the learning rule takes the form

$$
\begin{aligned}
R_{i k}(t+1)= & \eta\left(1-C_{\eta}+\psi C_{\eta}\right) \\
* & {\left[\left(_{C A 1} a_{i}(t)-\left(1-\psi C_{\theta}\right) \theta\right) * g\left({ }_{C A 3} a_{k}(t)\right)\right.} \\
& \left.\quad-\mu\left(C_{C A 1} a_{i}(t)-\left(1-\psi C_{\theta}\right) \theta\right) * R_{i k}(t)\right]+R_{i k}(t) .
\end{aligned}
$$

\section{Presentation of input patterns}

The capability of the network to display heteroassociative memory for different pairs of overlapping input patterns was studied. A small simulation was provided as an example (see Figs. 4, 5) of the learning of two pairs of input patterns. More extensive analysis was performed for a larger simulation examining the learning of five pairs of overlapping patterns (see Figs. 6, 7). For the large simulation, each pair involved simultaneous induction of (1) a specific pattern of activity in region CA3 $\left({ }_{C_{3} 3} a_{j}\right)$ containing six active neurons and (2) a specific associated pattern of activity in the entorhinal cortex $\left({ }_{\mathrm{EC}} a_{j}\right)$ containing six active neurons. The activity in entorhinal cortex influenced activity in region CAl via the synapses of $\mathrm{s} .1-\mathrm{m}\left(L_{i j}\right)$ and the activity in region CA3 influenced region CA1 activity via the synapses of $\mathrm{s}$. rad $\left(R_{i k}\right)$. Each entorhinal cortex pattern was presented in conjunction with one region CA3 pattern for a set number of simulation steps. After this set number of simulation steps, the presentation of the input pattern pair was halted, and another input pattern pair was presented. Different input pattern pairs were presented sequentially in this manner, as illustrated in Figures $4-7$, without any external setting of the dynamics of learning or recall. The internal feedback regulation of cholinergic modulation determined whether the network learned the pattern pair as new, or responded to it by recalling previously learned pattern pairs. In addition to simultaneous presentation of both input patterns in a pattern pair, during some simulation steps, the individual components of the input pattern pairs were presented without the associated pattern in the other region. Thus, input patterns were presented in three different configurations: (1) $\mathrm{CA} 3+\mathrm{EC}$ : patterns of activity induced in both entorhinal cortex and region CA3; (2) CA3 only: pattern of activity induced in region CA3 alone; (3) EC only: pattern of activity induced in the entorhinal cortex alone

When activity was induced in region CA 3 alone, activity would spread across the synapses of stratum radiatum into region $\mathrm{CA} 1$. The activity that resulted in region CAl was then compared with the different pat- 


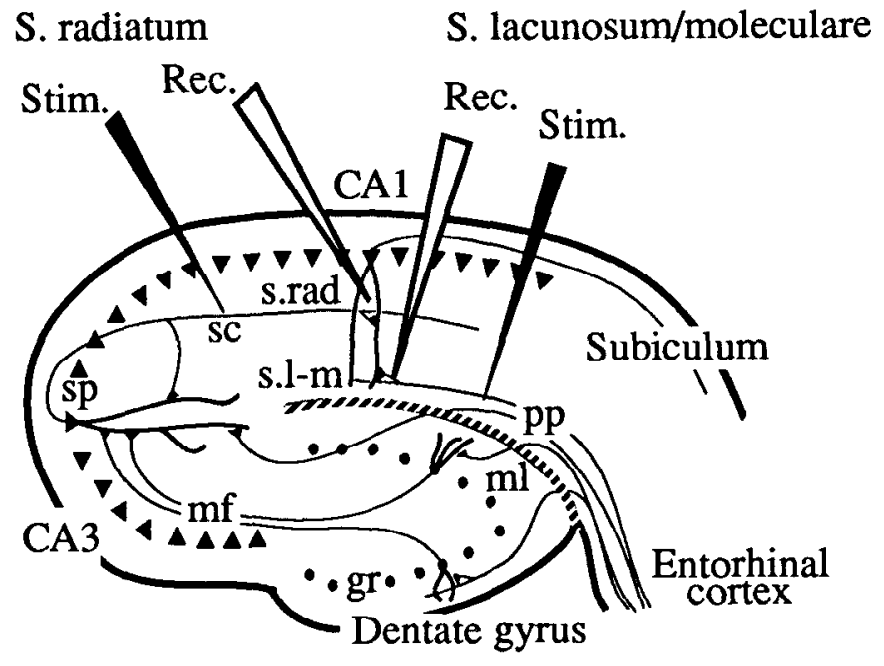

Figure 3. Experimental investigation of laminar differences in the cholinergic suppression of synaptic transmission in brain slice preparations of region CA1. Stimulating (Stim.) and recording (Rec.) electrodes are situated among the perforant path ( $p p$ ) fibers in stratum lacunosummoleculare $(s . l-m)$ and among the Schaffer collaterals $(s c)$ (and associational fibers) in stratum radiatum (s. rad). Stimulation was delivered alternately to either fiber layer, allowing analysis of the suppression of synaptic transmission in the same slice during the same perfusion. $s p$, stratum pyramidale; $m f$, mossy fibers; $g r$, stratum granulosum; $m l$, molecular layer. Broken line indicates location of cut to prevent activation of dentate gyrus molecular layer.

terns of activity that had been induced in the entorhinal cortex. Similarity between these patterns of activity was evaluated with a performance measure based on normalized dot products. This evaluation of activity patterns was performed across a full range of values for the maximal cholinergic suppression of synaptic transmission in $\mathrm{s} .1-\mathrm{m}$ and s. rad.

\section{Performance measure-normalized dot product}

The function of the network was evaluated using a performance measure consisting of the computation of a normalized dot product between the output pattern $A$ in region $\mathrm{CAl}$ and the target pattern $B$ corresponding to the associated input to CA1 from the entorhinal cortex (EC). For patterns $A$ and $B$, the normalized dot product takes the form

$$
\frac{A \bullet B}{\|A\|\|B\|}=\frac{\sum_{i=1}^{n} A_{i} * B_{i}}{\sqrt{\sum_{i=1}^{n} A_{i}^{2}} \sqrt{\sum_{i=1}^{n} B_{i}^{2}}} .
$$

The normalized dot product is equivalent to the cosine of the angle between the two vectors described by the two different patterns of activity. Thus, as the pattern of output in region CAl becomes more similar to the target input pattern from EC, the normalized dot product goes to 1 , and as the patterns of activity become completely nonoverlapping, the normalized dot product goes to 0 . This measure has been used previously to evaluate the function of simulations (Kohonen, 1988).

The performance measure was further modified to ensure that it accounted for the discriminability of different patterns of activity. In some cases, if the activity in region CA1 contained elements of all entorhinal cortex patterns, the normalized dot product with undesired patterns could be quite large. To measure whether the network was recalling one pattern well without recalling elements of other learned patterns, the normalized dot product of region CA 1 activity $A$ with all other stored patterns $C_{i}$ was subtracted from the normalized dot product of $A$ with the desired pattern $B$, as follows:

$$
P=\frac{A \bullet B}{\|A\|\|B\|}-\frac{1}{M} \sum_{i=1}^{M} \frac{A \bullet C}{\|A\|\left\|C_{i}\right\|}
$$

When no activity was elicited in region $\mathrm{CA} 1$, this measure was 0 . When activity in region $\mathrm{CA} 3$ elicited activity in region CA1 that overlapped strongly with the previously associated pattern in entorhinal cortex, without overlapping with other patterns, this performance measure would increase toward 1 . When activity in region CA 3 elicited $C A 1$ activity resembling all the patterns of entorhinal cortex activity, the performance measure went back to 0 . This performance measure has been used in previous simulations (Hasselmo et al., 1992; Hasselmo, 1994).

\section{Brain slice physiology}

The computational modeling presented above generated a specific prediction about the relative magnitude of the cholinergic suppression of synaptic transmission in $\mathrm{s}$. $\mathrm{l}-\mathrm{m}$ and $\mathrm{s}$. rad. This prediction was tested in brain slice preparations of rat hippocampal region CA1 (Fig. 3) by analysis of the influence of the cholinergic agonist carbachol on synaptic potentials elicited by stimulation of s. $1-\mathrm{m}$ and s. rad. These experiments utilized techniques developed for the comparison of $\mathrm{s}$. $\mathrm{l}-\mathrm{m}$ and $\mathrm{s}$. rad synaptic potentials in slice preparations (Colbert and Levy, 1992).

\section{Slice preparations}

Brain slices were prepared from 37 female albino Sprague-Dawley rats (Charles River Breeders, Inc.), 5-10 weeks of age, following techniques used in previous studies (Hasselmo and Bower, 1991, 1992; Barkai and Hasselmo, 1994a). After anesthetization with halothane, the rats were decapitated, and the brains were removed and immersed in chilled (4$6^{\circ} \mathrm{C}$ ), oxygenated, artificial cerebrospinal fluid (ACSF) with the following ionic composition (in mM): $\mathrm{NaHCO}_{3}, 26 ; \mathrm{NaCl}, 124 ; \mathrm{KCl}, 2.5 ; \mathrm{KH}_{2} \mathrm{PO}_{4}$, 1.2; $\mathrm{CaCl}_{2}, 2.4 ; \mathrm{MgSO}_{4}, 1.3$; and glucose, 10. The hippocampus was removed in a manner similar to that described previously (Alger et al., 1984). Slices $400 \mu \mathrm{m}$ thick were cut transverse to the septotemporal axis using a Stoelting tissue chopper. Slices were transferred to vials of ACSF continuously bubbled with a $95 \% \mathrm{O}_{2} / 5 \% \mathrm{CO}_{2}$ mixture for at least $1 \mathrm{hr}$.

Slices were placed on a nylon mesh in a standard submersion-type slice chamber perfused with $\operatorname{ACSF}\left(36 \pm 1.0^{\circ} \mathrm{C}\right)$ at $4 \mathrm{ml} / \mathrm{min}$. Slices were cut along the hippocampal fissure (between the dentate gyrus and region CA1) as described in previous research on $\mathrm{s}$. $1-\mathrm{m}$ (Colbert and Levy, 1992a,b; Levy and Colbert, 1992) to prevent responses from being evoked in the dentate gyrus and interfering with the responses recorded in s. 1-m (see Fig. 3). Slices were transilluminated to allow visually guided placement of stimulating and recording electrodes in both s. rad. and s. l-m. Rccording clcctrodes were glass micropipettes filled with $3 \mathbf{m}$ $\mathrm{NaCl}$ solution. Bipolar stimulating electrodes consisted of twisted strands of Teflon-insulated 0.002-inch-diameter platinum-iridium wire (A-M Systems, Inc.).

\section{Stimulation protocol}

Stimulation and recording of synaptic potentials was performed in both s. l-m and s. rad of a given slice during perfusion of carbachol, in order to control for the given conditions of the particular trial. Thus, each data point for $\mathrm{s}$. 1-m has a counterpart for $\mathrm{s}$. rad. A stimulating electrode was placed in each layer with a recording electrode about $300 \mu \mathrm{m}$ orthodromic to it. Electrodes were positioned as illustrated in Figure 3. In s. rad, the stimulating electrode was placed among the Schaffer collaterals at the center of the CA 1 region, approximately midway hetween the pyramidal cell layer and the superficial border of $\mathrm{s}$. 1-m (both of which are salient visual features). In $\mathrm{s}$. $1-\mathrm{m}$, the stimulating electrode was placed among the perforant path fibers toward the more distal half of $\mathbf{s}$ l-m (closer to the hippocampal fissure), in order to minimize the possibility of stimulating adjacent $\mathrm{s}$. rad fibers. EPSPs were required to have a minimum amplitude of $0.3 \mathrm{mV}$, without signs of a population spike.

Stimulus pulses were delivered under control of a Neurodata PG4000 digital stimulator. The stimulus pulses had a duration of $0.02 \mathrm{msec}$ and were delivered at $5 \mathrm{sec}$ intervals alternating between the two layers (each layer receiving stimulation every $10 \mathrm{sec}$ ). Evoked potentials were amplified using a custom-built extracellular amplifier and recorded using custom-written software on a Gateway $2000386 \mathrm{SX}$ computer. Averages of 10 successive digitized traces in a given layer were used in data analysis. In some cases, stimulus intensity was lowered during perfusion of bicuculline to prevent population spikes. Otherwise, stimulation amplitude and electrode placement were kept constant for the duration of an experiment.

A given preparation was considered stable when the amplitude of the EPSP did not change for at least a $10 \mathrm{~min}$ period. At this point, the 
perfusion bath was switched to a drug-containing ACSF solution. All drugs were obtained from Sigma Chemicals (St. Louis, MO). EPSPs were verified using a solution with low calcium $\left(200 \mu \mathrm{M} \mathrm{CaCl}_{2}, 8 \mathrm{~mm}\right.$ $\mathrm{MgSO}_{4}$ ) or TTX (1 mM solution administered directly).

\section{Drug perfusions}

All experiments were carried out in the presence of $20 \mu \mathrm{M}(-)$-bicuculline methiodide, in order to block the effect of GABAergic inhibitory interneurons. This technique was employed in previous studies of synaptic transmission in s. 1-m in CA1 by Colbert and Levy (1992a,b). This layer contains a significant number of feedforward inhibitory interneurons that run parallel to the perforant path fibers, and are stimulated simultaneously with them (Kunkel et al., 1988; Lacaille and Schwartzkroin, 1988). The evoked activity in these fibers results in an IPSP in the CA1 pyramidal cell dendrites in close temporal conjunction with the perforant path synaptic activation (Kunkel et al., 1988; Lacaille and Schwartzkroin, 1988), thereby obscuring the true height of the EPSP. Colbert and Levy (1992b) report that this $\mathrm{GABA}_{\mathrm{A}}$ inhibition strongly suppresses the EPSP evoked by perforant path stimulation, and that 20 $\mu \mathrm{M}$ bicuculline antagonizes the inhibition and can even cause CAl pyramidal cell firing in response to perforant path stimulation. In order to analyze more clearly the effects of carbachol on the perforant path EPSPs, the inhibitory (GABA ${ }_{A}$-mediated) responses were silenced. Unless mentioned otherwise, all solutions contained bicuculline.

The acetylcholine agonist carbachol (carbamyl choline chloride) was tested on slices in concentrations of $0.01,0.1,1.0,10,100$, and $500 \mu \mathrm{M}$. After stable baseline synaptic potentials had been obtained in both layers in control conditions, perfusion with the carbachol solution was initiated. Once carbachol reached its peak effect, the perfusion bath was switched back to the normal ACSF ( $20 \mu \mathrm{M}$ bicuculline) in order to begin a washout period and remove the carbachol from the perfusant. Carbachol was considered to have reached its peak effect when the (altered) size of the EPSP remained stable for at least 3 min following the onset of drug perfusion, or no effect was observed for $10 \mathrm{~min}$. A slice was never tested at the same drug concentration more than once, but some slices were tested at different concentrations in calculation of the doseresponse plot.

Several slices tested at $100 \mu \mathrm{M}$ carbachol were also perfused with 1.0 $\mu \mathrm{M}$ atropine, in order to assess the subtype of cholinergic receptor involved in mediating the cholinergic effects. In these experiments, the slice was first perfused with $100 \mu \mathrm{M}$ carbachol, washed to a stable potential, and then perfused with the atropine solution for $10 \mathrm{~min}$. After the atropine perfusion, the slice was perfused with a $100 \mu \mathrm{M}$ carbachol solution that contained $1.0 \mu \mathrm{M}$ atropine for at least $5 \mathrm{~min}$. EPSPs were recorded once they had stabilized during each period.

\section{Data analysis}

Extracellularly recorded signals were digitized for measurement of the exact value for the peak negative deflections of both the fiber volley and the EPSP of a given trace by manual placement of a cursor. In addition, the initial slope of each potential was calculated. The exact range used for slope calculation was manually selected as that between $25 \%$ and $75 \%$ of the EPSP amplitude, the region for which the slope was essentially linear. Both the EPSP slope and amplitude were evaluated in interpreting the effects of the particular perfusants on synaptic transmission.

\section{Results}

\section{Computational modeling of region $\mathrm{CA} 1$}

The simulation of region CA1 demonstrated that effective heteroassociative learning depended on selective cholinergic suppression of synaptic transmission in $\mathbf{s}$. rad, with weaker suppression or no suppression in s. 1-m (Figs. 4-7). The simulation showed the capability to sequentially learn or recall pairs of activity patterns arriving from region $\mathrm{CA} 3$ and the entorhinal cortex. Internal feedback regulation of cholinergic modulation within the simulation allowed the network to respond to unfamiliar pattern pairs with dynamics appropriate for learning, while responding to familiar pattern pairs with dynamics appropriate for recall. When presented with input in region CA3 alone, the network showed the capacity to recall the previously

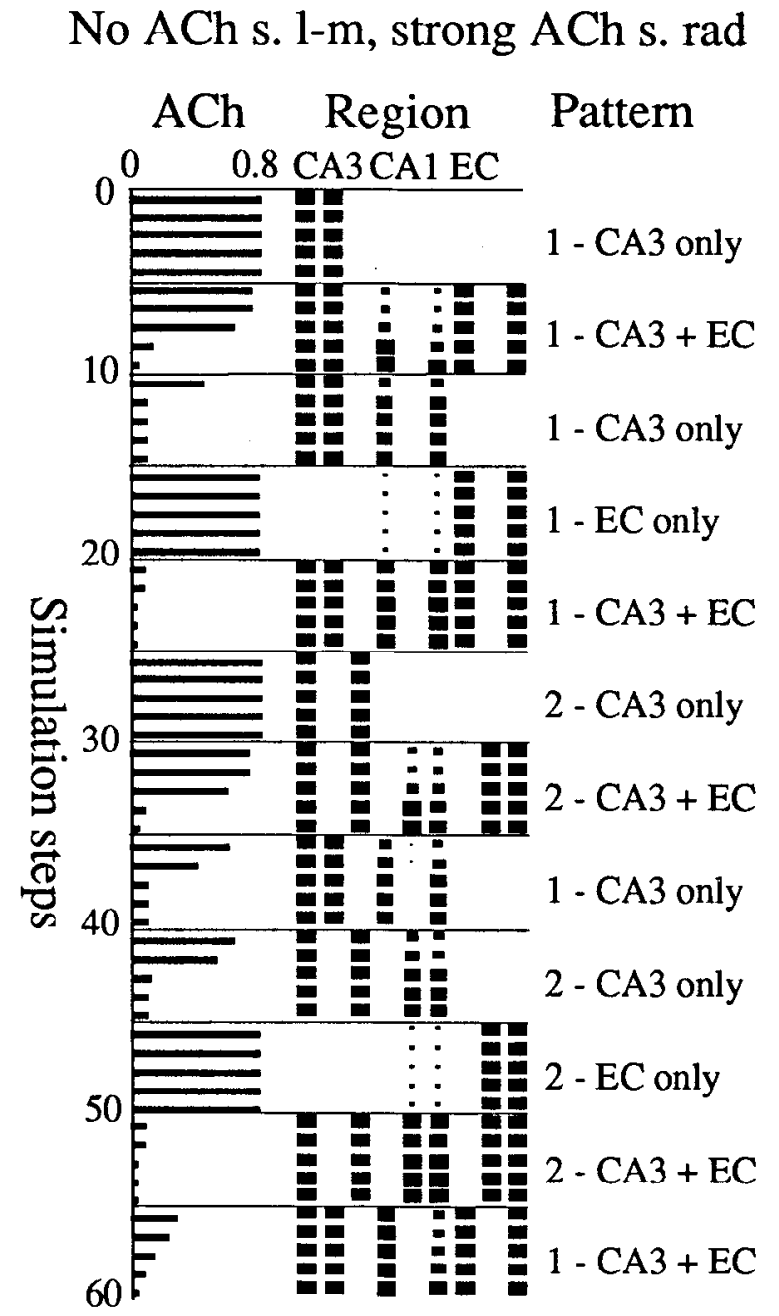

Figure 4. Strong cholinergic suppression in s. $\operatorname{rad}\left(C_{R}=0.8\right)$ coupled with no cholinergic suppression in $\mathrm{s}$. $1-\mathrm{m}\left(C_{L}=0.0\right)$ allows effective heteroassociative memory function in the simulation of region CA1. Neuronal output is shown across 60 simulation steps during sequential presentation of pairs of input patterns to a simple network with three neurons in each region. The center column shows the output of each of the three neurons in region $C A 3$, in region $C A 1$, and in the entorhinal cortex $(E C)$. Size of black squares indicates magnitude of output. In the left column, the level of cholinergic suppression of synaptic transmission in $\mathrm{s}$. rad is shown to vary between 0.8 and 0.0 depending upon feedback regulation from $\mathrm{CA} 1$ output. In the right column, the pair number for the input patterns is shown, along with an indication of which components of each pair are being presented. In steps $0-4$, presentation of the CA3 portion of pair 1 elicits no activity in region $C A 1$, and cholinergic suppression remains strong. In steps 5-9, presentation of both the $\mathrm{CA} 3$ and EC portion of pair 1 elicits activity in region CA 1 , causing strengthening of synapses in s. rad and a decrease in cholinergic modulation as the association is learned. In steps $10-14$, presentation of the CA3 portion alone now causes a decrease in cholinergic suppression, allowing recall of the activity in CAl previously induced by the associated input from EC. Note that during presentation of a second pair in steps 30-34, cholinergic suppression initially remains high, allowing the network to learn the new association without interference from the previous association (see Results for further description).

associated pattern of input from entorhinal cortex. As shown in Figure 7, computation of the performance measure in the network at a range of magnitudes of the maximal cholinergic suppression of synaptic transmission in s. 1-m and s. rad showed that the most effective learning occurred with strong suppression of synaptic transmission in $\mathrm{s}$. rad $\left(C_{R}\right.$ between 0.575 and 0.8$)$ 
and wcak or no suppression of synaptic transmission in s. $1-\mathrm{m}$ $\left(C_{L}\right.$ between 0.0 and 0.425$)$.

\section{Sequential heteroassociative learning of activity patterns}

Selective cholinergic suppression of synaptic transmission in s. rad but not in s. 1-m allowed the simulations to show effective heteroassociative memory function. As a simple example of the function of the network, Figure 4 illustrates the heteroassociative learning of activity patterns in a network with three neurons in each region. The center column of the figure shows the output of the three neurons in region $\mathrm{CA} 3$, in region $\mathrm{CA} 1$, and in the entorhinal cortex (EC) during each simulation step. The size of black squares represents the magnitude of the output of the neuron for that simulation step. As labeled on the right, the network was presented with different components of two pairs of input patterns. On the left, the magnitude of the cholinergic suppression of s. rad synapses is shown $\left(\mathrm{ACh}-\psi C_{R}\right)$. The maximal magnitude of cholinergic suppression in s. rad was $C_{R}$ $=0.8$ (allowing $20 \%$ of normal synaptic transmission strength). This magnitude changed in response to feedback regulation from region CAl as described above. The maximal magnitude of cholinergic suppression in $\mathrm{s}$. $1-\mathrm{m}$ was $C_{L}=0.0$. Thus, suppression was much stronger in $\mathrm{s}$. rad than s. $1-\mathrm{m}$. (The size of this smaller example simulation required some differences in simulation parameters: $H_{i l}=0.25, H_{i j}=0.2, H_{i k}=0.33, \eta=2, \mu=0.2, \xi=$ $3, v=1, \max R_{i k}=1.2, \min R_{i k}=0.05$.) The activity of the example shown in Figure 4 will be discussed for each set of simulation steps.

Steps $0-4$. In the first five simulation steps, the network was presented with only the CA3 portion of pattern pair 1 . This caused two neurons in region CA3 to show output, but the cholinergic suppression of synapses with random initial connectivity in s. rad prevented this output from causing activity in region CA1.

Steps 5-9. In the next five simulation steps, both portions of pattern pair 1 were presented, causing two neurons in region CA 3 and two neurons in entorhinal cortex to show output. The input from entorhinal cortex brings two neurons in region CAl up around firing threshold, and the input from region CA3 is sufficient to cause some output. Since $L_{i j}$ was the identity matrix, these two neurons matched the pattern of activity in EC. The learning rule then increased the strength of the $R_{i k}$ synapses between the active neurons in region CA 3 and the active neurons in region CAl. As the strength of these synapses increased, they increased the output of region CA1, until the CAl output was sufficient to decrease cholinergic modulation $(\psi)$. As cholinergic modulation decreased, the suppression of s. rad was removed, allowing strong output in region CA1.

Steps 10-14. The network was again presented with only the CA3 portion of pattern pair 1. Previously, this was insufficient to activate neurons in region $\mathrm{CA} 1$, but after the learning in steps 5-9 the strengthened synapses matched the activity in CA3 and were able to activate the associated activity pattern in region CAl. This initial input was sufficient to decrease cholinergic modulation, allowing the network to recall the previously associated pattern of entorhinal cortex activity.

Steps 15-19. Here the network was presented with only the EC portion of pattern pair 1 . Since the $s$. l-m synapses were weak and there was strong feedforward inhibition, this input alone only moderately activated the neurons in region CA1, causing insufficient activity to decrease the cholinergic modulation. Thus, the simulation showed the same weak influence of s. 1-m inputs on CA1 pyramidal cell activity as demonstrated in physiological experiments (Andersen et al., 1966; Yeckel and Berger, 1990; Colbert and Levy, 1992), but showed that even this weak input could be an important guiding influence when combined with input from CA3.

Steps 20-24. Repeated presentation of both components of pattern pair 1 showed that once the network had learned a paltern pair, it responded to that pattern pair as familiar. Note that cholinergic modulation was immediately turned off in response to this repeated presentation, allowing appropriate dynamics for recall.

Steps 25-29. The network did not perform recall in response to an unfamiliar pattern. Here, the CA3 portion of pattern pair 2 was presented. Although this CA3 activity overlapped with the CA 3 activity of pattern pair 1 , it did not sufficiently activate neurons in region CA1. Cholinergic modulation stayed high, and the network did not perform recall.

Steps 30-34. Presentation of both components of pattern pair 2 allowed learning of this pattern pair. Here selective cholinergic suppression of synaptic transmission was particularly important for associative memory function. Initially, for this unfamiliar pattern, cholinergic suppression of s. rad was high. This decreased the spread of activity across previously modified synapses in s. rad, allowing the input from entorhinal cortex to determine the pattern of activity. As the synapses of s. rad were strengthened, greater output in region CA1 decreased the cholinergic modulation, allowing greater activity. Thus, cholinergic suppression in s. rad prevented possible interference from previous associations until the new information was learned accurately.

Steps 35-39. After learning of pattern pair 2, presentation of the CA3 portion of the previously learned pattern pair 1 directly evoked the previously associated pattern of EC input.

Steps 40-44. Subsequent presentation of the CA3 portion of pattern pair 2 evoked the associated pattern of EC input for pattern pair 2.

Steps 45-49. Presentation of only the EC portion of pattern pair 2 caused little output from the pyramidal cells of region CA1.

Steps 50-54. Presentation of the familiar pattern pair 2 immediately suppressed cholinergic modulation, decreasing learning in response to this familiar pattern pair.

Steps 55-59. Presentation of the familiar pattern pair 1 also suppressed cholinergic modulation. At this point, it could be said that the network recognized the two learned input pattern pairs.

\section{Feedback regulation of selective cholinergic suppression for learning and recall}

As shown in Figure 4, feedback regulation of cholinergic modulation allowed the network to internally determine appropriate dynamics for learning or for recall. If the pattern presented to region CA3 was part of a previously learned pattern pair, then the initial response in region CAl would be strong, decreasing cholinergic modulation and allowing strong recall of the previously associated pattern of activity. If the pattern presented to region CA3 was not part of a previously learned pattern pair, then the initial response in region CAl would be weak, and cholinergic modulation would maintain the appropriate dynamics for learning the new pattern until sufficient synaptic modification allowed the network to shut down cholinergic modulation. 
The laminar selectivity of the cholinergic suppression of synaptic transmission was important for setting the appropriate dynamics for learning and recall. As shown in Figure 4, with strong cholinergic suppression of synaptic transmission in s. rad, the pattern of activity in entorhinal cortex set the pattern of postsynaptic activity in region CA1, while the presynaptic activity at connections $R_{i k}$ was determined by the pattern in region CA3. For unfamiliar pattern pairs, this allowed clean storage of an association between the individual pairs of patterns. When the pattern presented was familiar, suppression of synaptic transmission was decreased by feedback regulation, and presentation of patterns of activity to region CA3 elicited the associated pattern of activity in region CAl with little interference from other associations.

Different relative magnitudes of cholinergic suppression caused problems with performance, as shown in Figure 5 for the initial part of the same pattern sequence presented in Figure 4. Figure $5 A$ illustrates the breakdown of function with weak cholinergic suppression of synaptic transmission in $\mathrm{s} . \operatorname{rad}\left(C_{R}=0.4\right)$ coupled with no suppression of synaptic transmission in $\mathrm{s} .1-\mathrm{m}\left(C_{L}=\right.$ 0.0 ). With weak cholinergic suppression of synaptic transmission in s. rad, synaptic transmission at the Schaffer collaterals altered the pattern of postsynaptic activity in region CAl, altering the pattern determined by entorhinal cortex input. This recall of previously stored associations during learning of new patterns caused the network to show poorer performance. Effects at individual simulation steps are discussed below.

Steps 0-4. With weak cholinergic suppression of synaptic transmission in $\mathrm{s}$. rad, presentation of the CA3 portion of pattern pair 1 caused activity to immediately spread along the synapses into region CA1. Since these synapses had random initial connectivity, they induced a random, distributed pattern of activity in region CA1. Synaptic modification then strengthened an association between this activity and the activity in region CA3. (Here s. rad synapses are the predominant influence on postsynaptic activity, thereby causing them to undergo self-organization.)

Steps 5-9. Subsequent presentation of both portions of pattern pair 1 could not set the pattern of activity in CAl to the EC input.

Steps 10-14. Even after presentation of both components of the pattern, presentation of the $\mathrm{CA} 3$ component activated region $\mathrm{CA} 1$ in a distributed manner.

Steps 15-34. Similar difficulties occurred during subsequent simulation steps.

Figure $5 B$ shows the breakdown in function with strong cholinergic suppression in s. $1-\mathrm{m}\left(C_{L}=0.8\right)$ along with strong cholinergic suppression in $\mathrm{s}$. $\mathrm{rad}\left(C_{R}=0.8\right)$. Here the synaptic transmission in $\mathrm{s}$. 1-m was not sufficiently strong to guide the learning of associations in region CAl.

Steps 0-4. With strong cholinergic suppression of synaptic transmission in s. rad, presentation of the CA3 portion of pattern pair 1 did not induce activity in CA1.

Steps 5-9. In contrast to Figure 4, even when both portions of pattern pair 1 were presented, cholinergic suppression in both s. $1-\mathrm{m}$ and $\mathrm{s}$. rad prevented sufficient activity from developing in region CAl. In this case, very little synaptic modification occurred, and cholinergic modulation remained strong.

Steps 10-14. Subsequent presentation of the CA3 portion of pattern pair 1 did not elicit the associated pattern of EC activity.

Steps 15-34. Similar difficulties occurred for subsequent simulation steps.

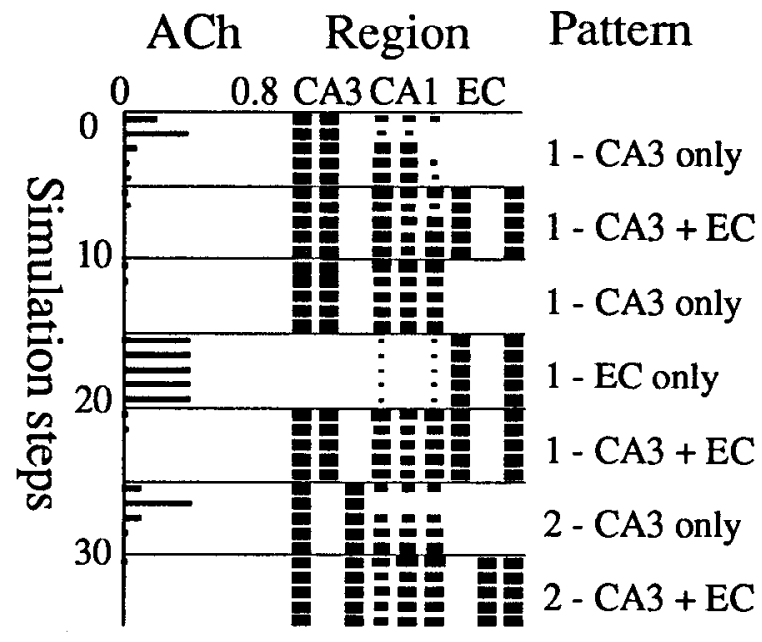

B. Strong ACh s. 1-m, strong ACh s. rad

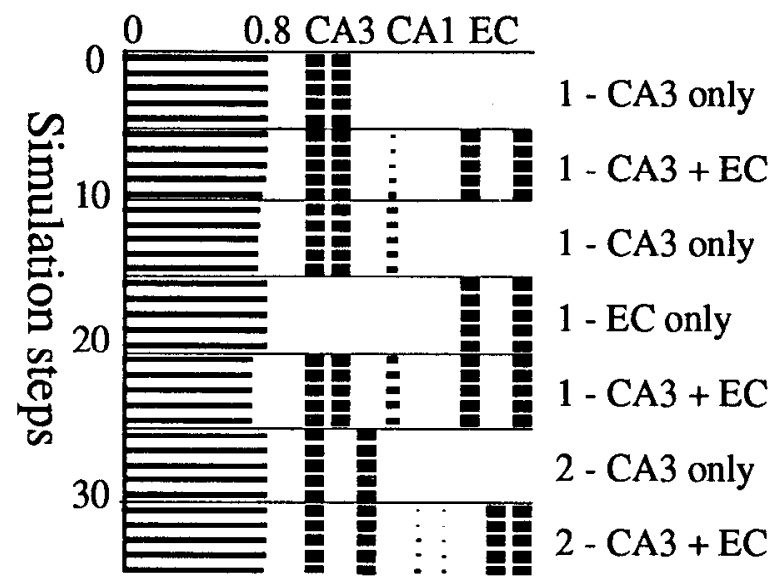

Figure 5. A, Heteroassociative memory function breaks down with weak cholinergic suppression in $\mathrm{s}$. $\operatorname{rad}\left(C_{R}=0.4\right)$ and no suppression in s. $1-\mathrm{m}\left(C_{L}=0.0\right)$. All other parameters are the same as for the simulation in Figure 4. Neuron output is shown across 35 simulation steps during sequential presentation of the same patterns as in Figure 4. Note that weak suppression in s. rad allows synaptic transmission in s. rad to interfere with effective associative memory function. In steps $0-4$, with presentation of the CA3 portion of pair 1 , activity spreads into region $\mathrm{CA} 3$, causing synaptic modification and suppression of cholinergic modulation, despite the lack of any input to be associated. In steps 5-9, during presentation of both parts of pair 1, synaptic transmission at previously modified synapses interferes with learning of the association. Similar interference occurs during presentation of pair 2 in steps 30-34 (see Results). B. Memory function also breaks down with strong cholinergic suppression in both s. $1-\mathrm{m}\left(C_{L}=0.8\right)$ and $\mathrm{s} . \operatorname{rad}\left(C_{R}\right.$ $=0.8$ ). In this case, strong suppression in $\mathrm{s}$. $1-\mathrm{m}$ prevents activity in EC from setting the activity to be learned in CAl. In steps 5-9, during presentation of both parts of pair 1 , very little activity is induced in region $\mathrm{CAl}$, preventing the modification of synapses in $\mathrm{s}$. rad and allowing cholinergic suppression to remain strong (see Results for further description).

Performance at a range of magnitudes of suppression in $s . l-m$ and s. rad

Selective cholinergic suppression of synaptic transmission in s. rad but not s. l-m was essential for effective heteroassociative memory function in a larger network simulation of region CA1. This larger simulation contained 30 neurons in each region and was tested on the storage of five pairs of overlapping input patterns. These pairs of overlapping input patterns were pre- 
No ACh s. 1-m, weak ACh s. rad.

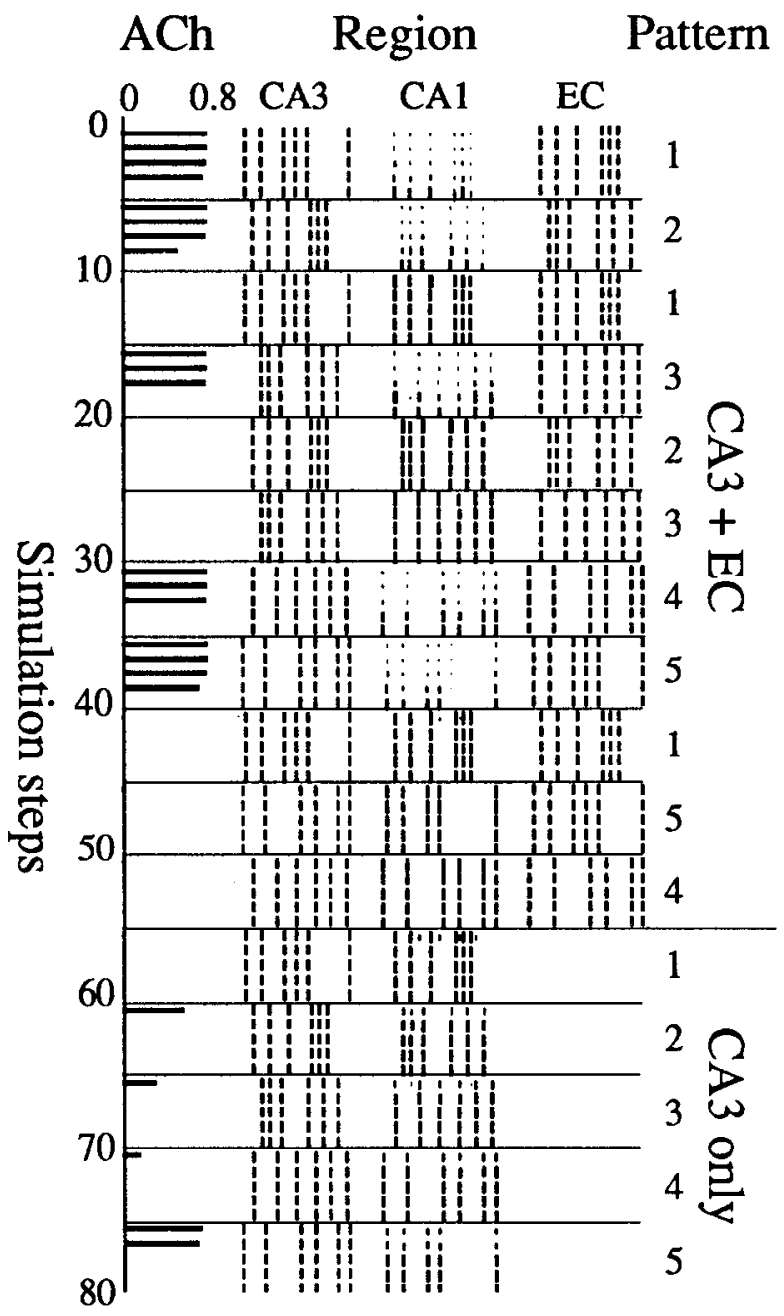

Figure 6. In a larger simulation of region CAl with storage of more pairs of input patterns, strong cholinergic suppression in $\mathrm{s}$. $\operatorname{rad}\left(C_{R}=\right.$ 0.8 ) coupled with no cholinergic suppression in s. 1-m $\left(C_{L}=0.0\right)$ still allows effective heteroassociative memory function. Neuronal output is shown across 80 simulation steps in a network with 30 neurons in each region during sequential presentation of five pairs of input patterns with six active neurons in each pattern. Here the network is presented twice with each pair of input patterns. As seen in the left column $(A C h)$, the cholinergic suppression of synaptic transmission in $\mathbf{s}$. rad is high during initial presentation of novel input pattern pairs 1 and 2 and decreases as these patterns are learned. Subsequent presentation of the familiar pattern pair 1 decreases cholinergic suppression, allowing recall. Across subsequent simulation steps, cholinergic modulation increases for presentation of novel pairs, and is decreased during presentation of familiar pairs. In the last five presentations, only the CA 3 portion of each pattern pair is presented. In these cases, the network accurately recalls the previously associated input pattern from entorhinal cortex $(E C)$. Computation of the performance measure for these last five presentations yields a performance measure of 0.86 .

sented sequentially, as shown in Figure 6, with a final evaluation of recall performance during sequential presentation of only the CA3 portion of each of the five pairs of patterns.

Figure 6 shows the function of the larger simulation with strong suppression in $\mathrm{s}$. $\mathrm{rad}\left(C_{R}=0.8\right)$ and no suppression in $\mathrm{s}$. $1-\mathrm{m}\left(C_{L}=0.0\right)$. The layout of this figure is the same as in Figures 4 and 5 , except that each region has a larger number of neurons. The cholinergic suppression of $s$. rad is shown on the left. Note

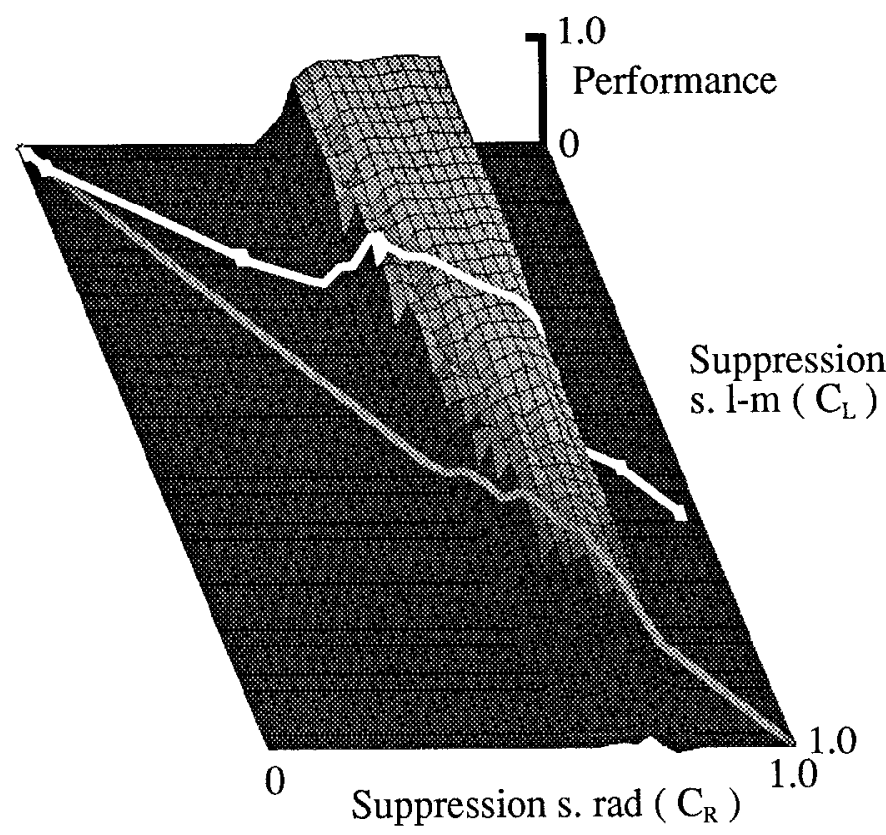

Figure 7. Performance measure of heteroassociative memory function across a full range of levels of the maximal cholinergic suppression of synaptic transmission in s. 1-m $\left(C_{L}=0.0-1.0\right)$ and s. rad $\left(C_{R}=0.0\right.$ 1.0), with 40 levels tested for each layer. Each of the 1600 data points in this plot represents results from a simulation such as that shown in Figure 6 , with the difference between the simulations being the level of cholinergic suppression in $\mathrm{s}$. $1-\mathrm{m}$ and $\mathrm{s}$. rad. The $z$-axis shows the value of the performance measure $P$ computed for each simulation during the last five presentations (CA3 only), with higher (better) values of $P$ shown with lighter shading. The magnitude of the maximal suppression of synaptic transmission is plotted on the $x$-axis for s. rad and on the $y$ axis for s. $1-\mathrm{m}$. The best performance $(P>0.8)$ appears with strong maximal cholinergic suppression in s. $\operatorname{rad}\left(C_{R}=0.575-0.8\right)$ and weak maximal suppression in s. $1-\mathrm{m}\left(C_{L}=0.0-0.425\right)$. For low values of $C_{R}$ (left side, $C_{R}<0.5$ ), interference during learning causes the network to respond to all CA3 patterns with broadly distributed activity in CA1, giving low performance values. For high values of $C_{L}$ (bottom portion, $C_{L}>0.6$ ), insufficient EC input prevents accurate learning of associations, giving low performance values. For very high values of $C_{R}\left(C_{R}\right.$ $>0.8$ ), suppression of $\mathrm{s}$. rad prevents sufficient activity in CAl to decrease cholinergic suppression and allow recall. Gray line represents unity $\left(C_{L}=C_{R}\right)$. White line represents the dose-response plot from experimental data (Fig. 10) plotted on these coordinates.

that as each new pair of input patterns is presented, the network responded appropriately dependent upon the novelty or familiarity of the pair. For novel patterns, cholinergic suppression remained high, and the network learned the pattern as a new association. For familiar patterns, cholinergic suppression decreased, and the network recalled the pattern as a previous association. At the bottom of the figure, when only the CA3 portion of each pair was presented, the network responded to each pattern with the associated $\mathrm{EC}$ input pattern, with no interference between patterns, and only one missing component. The performance measure was computed for the last five presentations (CA3 only). For each CA3 input pattern, the normalized dot product was computed for the activity in region CAl compared with the previously associated EC input pattern. In addition, the average normalized dot product of region $\mathrm{CAl}$ activity with other EC input patterns was subtracted from this measure. For the magnitudes of cholinergic suppression shown in Figure 6 , the performance measure was 0.86 , reflecting the 
fact that the network effectively recalled all of the EC input patterns except for one component in pattern pair 5 .

The performance measure was computed across the full range of magnitudes of cholinergic suppression in $\mathrm{s} .1-\mathrm{m}$ and in $\mathrm{s}$. rad, as shown in Figure 7 . With the same initial conditions, the network was tested at magnitudes ranging from 0.0 to 1.0 for both $C_{L}$ and $C_{R}$. For each magnitude, the network was presented sequentially with the full range of pairs of input patterns, as shown in Figure 6 . The recall of the network was evaluated by computing the performance measure $P$ for CA1 activity in response to the CA3 portion of each pattern pair. The performance measure $P$ is plotted on the z-axis in Figure 7, with cholinergic suppression of synaptic transmission in $\mathrm{s}$. rad on the $\mathrm{x}$-axis and suppression of synaptic transmission in $\mathrm{s}$. 1-m on the $y$-axis. Higher performance measure values have lighter shading. Note that for most values of cholinergic suppression, the performance was quite poor $(0.0)$. The best performance $(P>0.8)$ was obtained with strong maximal cholinergic suppression in $\mathbf{s}$. rad $\left(C_{R}=0.575-0.8\right)$ and weak maximal suppression in $\mathrm{s}$. $1-\mathrm{m}\left(C_{L}\right.$ $=0.0-0.425)$. On the left, when suppression in s. rad was weak, the network responded with broadly distributed activity in region CA1. The recall of all patterns caused a low performance value. On the right, when suppression in s. rad was too strong, the network could not generate sufficient activity in region CA1 to decrease cholinergic modulation and allow recall. With little activity in region $\mathrm{CA} 1$, the performance measure was low. Note that good performance occurred only when suppression in $\mathrm{s}$. $l-m$ was weaker than suppression in $s$. rad. That is, the good performance (lighter shading) appears only above the gray line marking equal suppression in both layers $\left(C_{L}=C_{R}\right)$. Performance was consistently highest when suppression in $\mathrm{s}$. $1-\mathrm{m}$ was considerably weaker than suppression in s. rad. For values where maximal suppression in $\mathrm{s}$. 1-m was larger than maximal suppression in $\mathrm{s}$. rad (bottom of plot), the suppression of synaptic transmission in s. 1-m prevented the input from the entorhinal cortex from setting activity in region CA1 such that an association could be learned accurately. Thus, the results from the simulation predict that for the best heteroassociative memory function within the network, region CA1 of the hippocampal formation should demonstrate strong cholinergic suppression of synaptic transmission in s. rad and relatively weaker cholinergic suppression of synaptic transmission in s. 1-m.

In Figure 7, the white line shows the dose-response plot obtained in the experimental work for the effect of cholinergic modulation on synaptic transmission in s. rad and s. 1-m. Note that the plot of experimental data crosses the region of maximal performance. This plot will be discussed further in the following section.

\section{Brain slice physiology}

Carbachol effects

As predicted by the simulation, experimental results demonstrated that the cholinergic suppression of synaptic transmission was stronger in stratum radiatum than in stratum lacunosummoleculare. Perfusion of the cholinergic agonist carbachol (100 $\mu \mathrm{M})$ consistently caused a greater decrease in the slope and peak amplitude of the EPSP in s. rad. than of the EPSP in s. $1-\mathrm{m}$. This difference in suppression appeared consistently for EPSPS recorded in the same slice during the same perfusion. However, there was some suppression of the EPSPs recorded in both layers. These results are illustrated in sequences of recordings taken during $100 \mu \mathrm{M}$ carbachol trials (Fig. 8). The mean rising slope
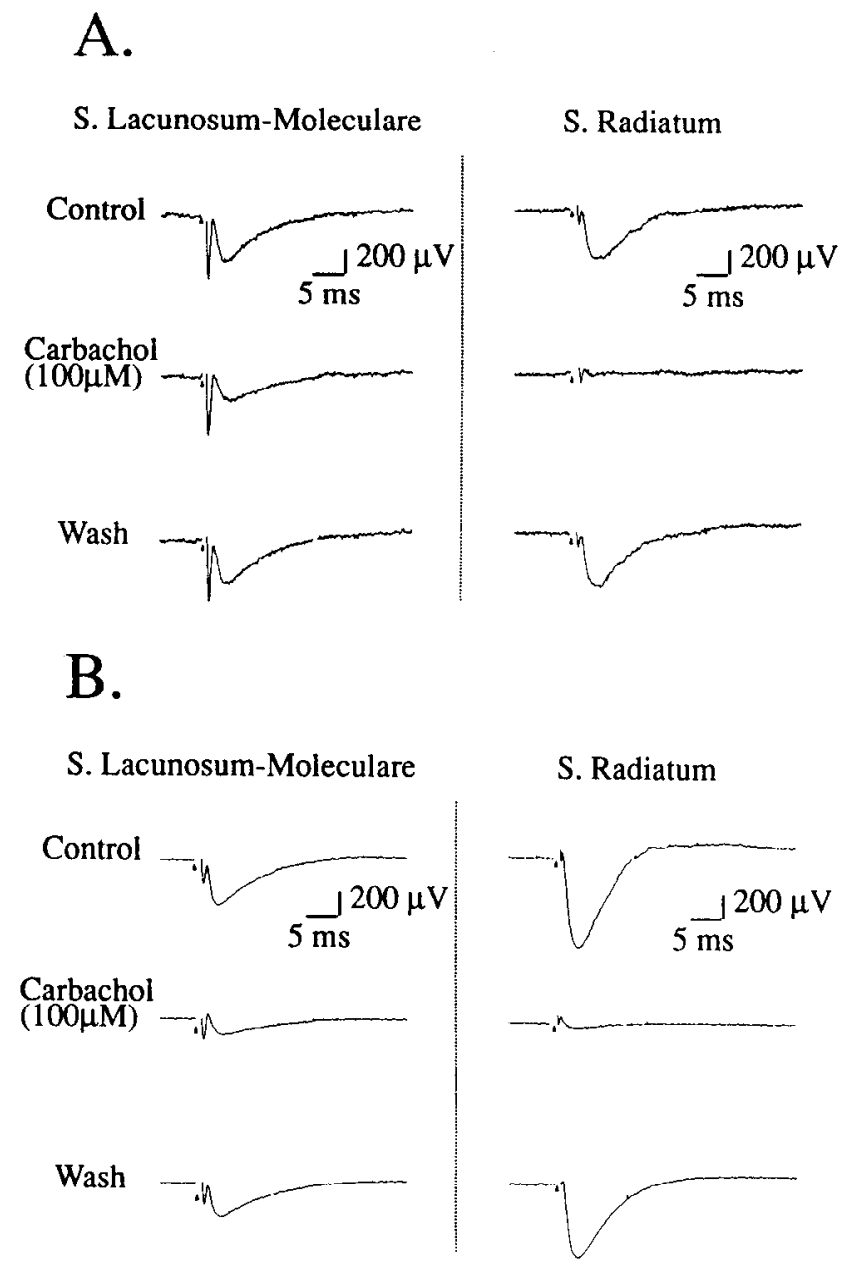

Figure 8. The cholinergic agonist carbachol suppresses synaptic potentials stimulated and recorded in stratum radiatum (s. rad) more strongly than synaptic potentials stimulated and recorded in stratum lacunosum-moleculare (s. $1-\mathrm{m})$ of the same slice during the same experiment. The differential effect of the cholinergic agonist carbachol is shown for two different experiments. Recordings in the two layers were taken before, during, and after perfusion of $100 \mu \mathrm{M}$ carbachol. $A$, In this slice, the slope of the s. rad EPSP was suppressed by $90.7 \%$, while the slope of the s. 1 -m EPSP was suppressed by only $46.0 \%$ during the same perfusion. $B$, In this slice, the slope of the s. rad EPSP was suppressed by $87.6 \%$, while the slope of the s. 1-m EPSP was suppressed by only $54.6 \%$. Fiber potentials remained stable during carbachol perfusion in both layers in both slices. Potentials recovered after 20 min of ACSF wash.

of the EPSP was decreased by a greater amount in the recordings from $s$. rad $[89.1 \pm 3.0 \%( \pm \mathrm{SE})$ reduction] than in the recordings obtained in the same slices from s. $1-\mathrm{m}(40.1 \pm 4.1 \%)$. Similarly, the mean suppression of the peak EPSP amplitude in $\mathrm{s}$. rad at this concentration of carbachol was $87.6 \pm 1.81 \%(n=13)$, while the mean suppression of the EPSP amplitude recorded in s. $1-\mathrm{m}$ during the same set of experiments was only $53.1 \pm 3.92 \%$ $(n-13)$. A paired $t$ test demonstrated significant differences between the two layers in the cholinergic suppression of both EPSP amplitude $(t=7.46, \mathrm{df}=12 ; p<0.0001)$ and slope $(t=$ $11.7, \mathrm{df}=12 ; p<0.0001)$. The results obtained from the 100 $\mu \mathrm{M}$ carbachol perfusions are summarized in Figure 9. The differential effect of carbachol was consistent for all slices tested, although there was a variation between slices in the exact percentages. The carbachol effect was also reversible in both layers, requiring approximately $15-30 \mathrm{~min}$ to achieve maximum wash. 

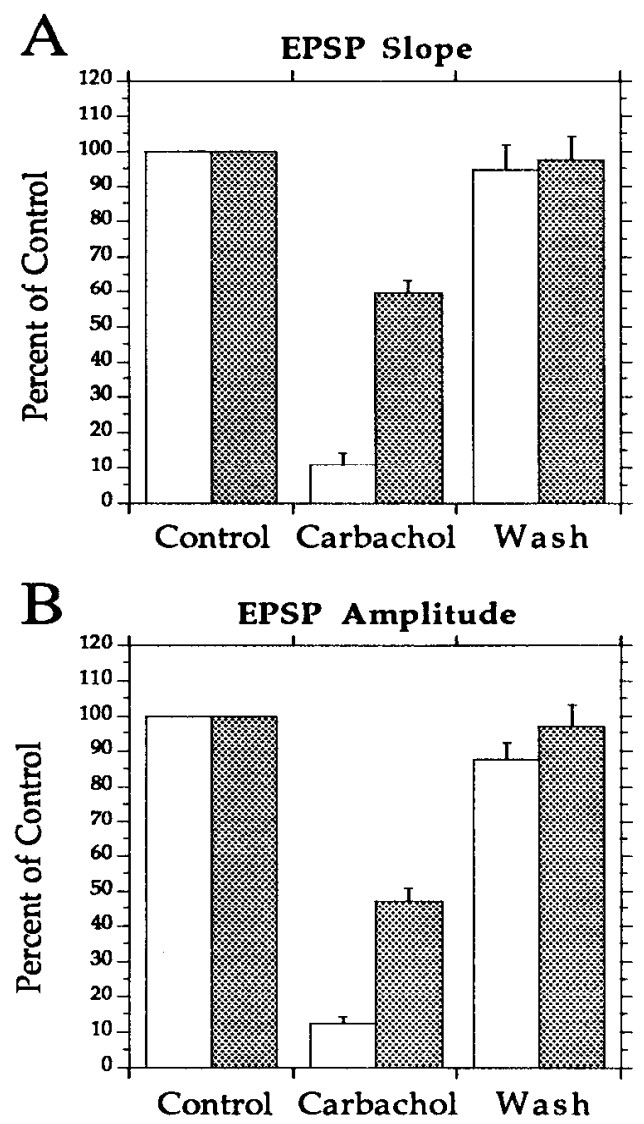

Figure 9. Summary of the mean effect of $100 \mu \mathrm{M}$ carbachol on EPSP slope $(A)$ and EPSP amplitude of synaptic potentials $(B)$ recorded in stratum radiatum (s. rad, open columns) and in stratum lacunosummoleculare (s. $1-\mathrm{m}$, shaded columns). Slope and amplitude of potentials are plotted as mean percentage of control response during control conditions, during perfusion of $100 \mu \mathrm{M}$ carbachol, and after washout of carbachol. Error bars represent SE. In 13 experiments for each layer, both the EPSP slope and amplitude were suppressed more strongly in s. rad than in s. 1-m. EPSP slope shows a slightly greater differential effect in response to carbachol than EPSP amplitude. ACSF washes restored both the slopes and amplitudes to approximately their former levels.

In both of these layers, the fiber potential was relatively unaffected, decreasing by a mean of $17.6 \%$ in s. rad and $16.4 \%$ in s. l-m during perfusion with $100 \mu \mathrm{M}$ carbachol.

The effects of perfusion with carbachol were tested at concentrations ranging from $0.01 \mu \mathrm{M}$ to $500 \mu \mathrm{M}$ with concurrent recording from both $\mathrm{s}$. rad and s. $1-\mathrm{m}$. For each concentration, experiments in five slices were performed, except for $100 \mu \mathrm{M}$, for which 13 experiments were performed. The suppression of synaptic transmission was significantly different between the layers at concentrations of $10 \mu \mathrm{M}$ or larger. The dose-response plot for the effects of carbachol on the height of synaptic potentials in both layers of CA1 is shown in Figure 10. The $\mathrm{IC}_{50}$ for carbachol was estimated by interpolating between the data points on either side of $50 \%$ suppression for the effect measured in cithcr laycr, yiclding an $\mathrm{IC}_{50}=7.33 \mu \mathrm{M}$ for s. $\mathrm{rad}$ and $\mathrm{IC}_{50}=$ $89.89 \mu \mathrm{M}$ for s. $1-\mathrm{m}$. The difference in these values reflects the fact that a considerably higher concentration of ACh would be required for the same influence on synaptic transmission in s. $1-\mathrm{m}$ as opposed to $\mathrm{s}$. rad.

For comparison with the model, the dose-response was plotted on the performance measures plotted for different magni-

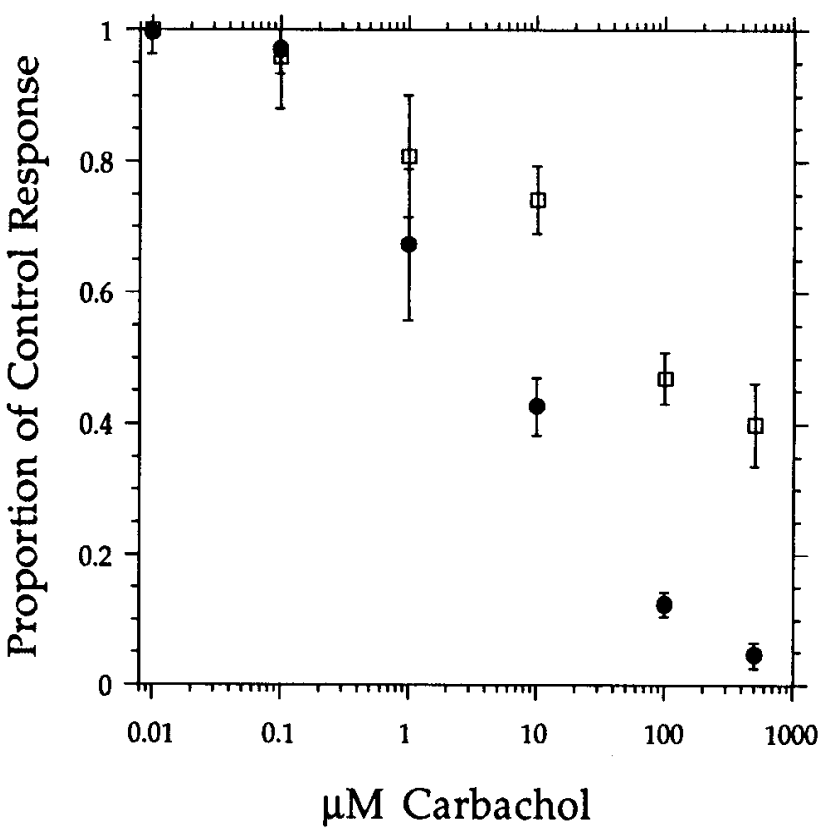

Figure 10. Dose-response plot showing the effect of carbachol in stratum lacunosum-moleculare (s. $1-\mathrm{m}, \square$ ) and stratum radiatum (s. rad, $\bullet$ ) of region CAl at a full range of concentrations from $0.1 \mu \mathrm{M}$ to $500 \mu \mathrm{M}$. The proportion of the EPSP amplitude conserved is on the $y$-axis (percentage of mean baseline response), while the concentrations are plotted logarithmically on the $x$-axis. Standard error bars are included for each mean response. Differential effects of carbachol application are most prominent at doses above $1 \mu \mathrm{M}$, where the two dose-response plots diverge from one another. This data is also plotted in Figure 7.

tudes of suppression in the simulation (Fig. 7). As can be seen in Figure 7, the dose-response plot is in the upper portion of the graph, reflecting the weaker suppression in $\mathrm{s}$. $1-\mathrm{m}$ than $\mathrm{s}$. $\mathrm{rad}$, and the experimental data crosses a region of good performance in the model. Note that the data points in the experimental dose-response plot are slightly different than the points in Figure 7, since Figure 7 shows performance for different values of maximal cholinergic suppression. This suggests that if the parameters of the simulation adequately reflect the parameters of cortical function, then the maximal ambient levels of $\mathrm{ACh}$ in region C.A1 might be just over $10 \mu \mathrm{M}$. These concentrations of the dose-response plot would correspond to a region with performance measure values over 0.8 in Figure 7.

\section{Muscarinic antagonism of cholinergic suppression}

The effect of carbachol appears to be mediated by muscarinic ACh receptors, since perfusion with the specific muscarinic antagonist atropine for $10 \mathrm{~min}$ prior to carbachol perfusion blocks the cholinergic effects in both layers (see Fig. 11). In each of these slices $(n=5)$, a $100 \mu \mathrm{M}$ carbachol suppression was performed and washed prior to testing atropine at a concentration of $1.0 \mu \mathrm{M}$. In all of these slices, carbachol had a characteristic effect prior to atropine perfusion, but had no suppressing effect after atropine perfusion. These data are summarized in Figure 12.

Atropine itself did not appear to have any consistent effect on the EPSPs after washout of carbachol, although in several cases it raised the EPSP from a partially successful wash (of $\sim 85-90 \%$ of control amplitude) to a full return of the control signal. This implies that there may be a sustained effect of carbachol which does not always wash fully under normal condi- 


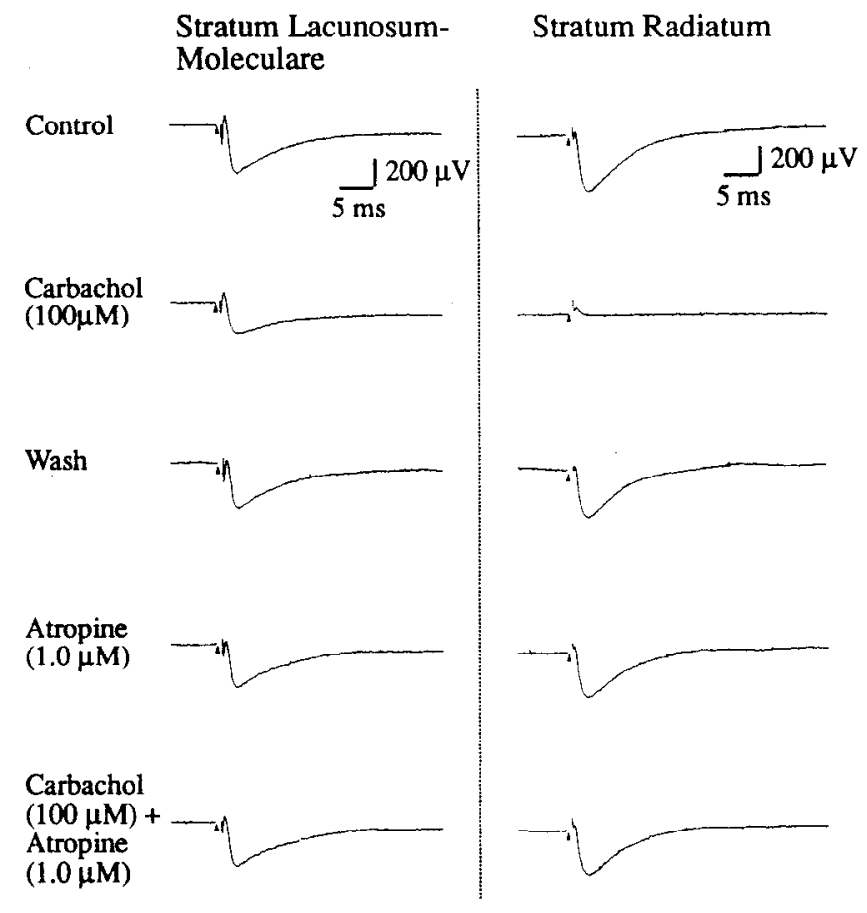

Figure 11. Atropine blocks the suppression of synaptic transmission by carbachol. $A$, During recording from both $\mathrm{s}$. $1-\mathrm{m}$ and $\mathrm{s}$. rad in the same slice, synaptic potentials were recorded before, during, and after perfusion with carbachol $(100 \mu \mathrm{M})$ to show the selective cholinergic suppression of synaptic transmission. After this effect was washed, the slices were perfused with $1.0 \mu \mathrm{M}$ atropine for $10 \mathrm{~min}$, and then perfused with a solution containing both $1.0 \mu \mathrm{M}$ atropine and $100 \mu \mathrm{M}$ carbachol. Atropine blocked the effects of carbachol on synaptic transmission in both layers, indicating that cholinergic suppression in both layers is mediated by muscarinic ACh receptors.

tions. The effect of carbachol during atropine perfusion was insignificant. In $1.0 \mu \mathrm{M}$ atropine, perfusion of $100 \mu \mathrm{M}$ carbachol resulted in a mean $0.31 \pm 1.69 \%$ increase in the EPSP amplitudes in $\mathrm{s}$. $1-\mathrm{m}$ and a suppression of $1.2 \pm 6.67 \%$ in $\mathrm{s}$. rad. This compares to a mean suppression induced by carbachol before atropine (in the same slices) of $51.3 \%$ and $90.4 \%$ for $\mathrm{s} .1-\mathrm{m}$. and s. rad, respectively. Paired $t$ tests on the EPSP peaks measured in atropine and during carbachol after atropine yielded no significant difference between the two groups. The slight shift in responsiveness over the later portions of these graphs appeared to be due to gradual changes in experimental conditions rather than a specific effect of atropine.

\section{Bicuculline}

All of the above experiments were conducted in the presence of $20 \mu \mathrm{M}$ bicuculline. Because of its ubiquitous use in this series of experiments, the effects of bicuculline on the recorded potentials were documented. In these experiments, the field potentials were allowed to stabilize for a long period before perfusion with bicuculline and were compared to the potentials recorded in bicuculline. In seven out of 12 such cases, bicuculline administration caused the stimulation of $s$. rad to evoke population spike activity. This spiking was either a burst (several population spikes) or it did not occur at all. The EPSP slope increased an average of $160.9 \pm 111.1 \%$ in $\mathrm{s} .1-\mathrm{m}$ in response to bicuculline, while the slope of s. rad responses increased by only $25.8 \pm 7.0 \%$. The EPSP amplitudes recorded in s. 1-m were increased by $89.0 \pm 30.2 \%$, and these could be reliably measured

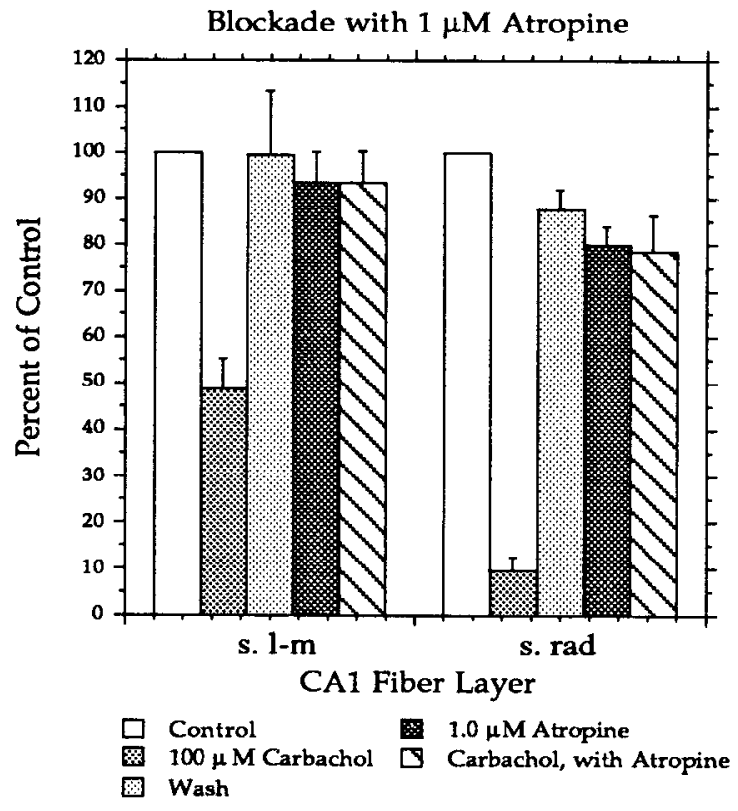

Figure 12. Summary of the blockade of suppression of synaptic transmission by $1.0 \mu \mathrm{M}$ atropine $(n=5)$. The height of synaptic potentials as percentage of control response is shown for control conditions, during perfusion of $100 \mu \mathrm{M}$ carbachol (showing suppression of synaptic transmission), after washout of carbachol, during perfusion of $1 \mu \mathrm{M}$ atropine, and during perfusion of both $1 \mu \mathrm{M}$ atropine and $100 \mu \mathrm{M}$ carbachol (showing blockade of suppression). Effects in stratum lacunosum-moleculare $(s . l-m)$ are shown on the left, and effects in stratum radiatum (s. rad) are shown on the right.

because perforant path stimulation did not evoke population spikes at any of the stimulation values used. The mean control EPSP amplitude for s. rad was $-0.683 \mathrm{mV}$ in slices where perfusion with bicuculline evoked population spiking, while the mean was $-0.378 \mathrm{mV}$ in slices where perfusion did not result in any population spiking. The fiber volleys in both layers did not increase, decreasing $1.8 \pm 3.6 \%$ in $\mathrm{s} .1-\mathrm{m}$ and $19.9 \perp 13.1 \%$ in s. rad.

\section{Controls}

Several slices $(n=4)$ were perfused with a low-calcium/highmagnesium ACSF in order to verify the EPSP signals. In all trials, the low-calcium solution abolished the EPSP component of both the s. rad and s. 1-m signals without significantly affecting the magnitude of the fiber volley. This was taken as evidence that the EPSP components of the signals were indeed reflecting synaptically mediated events. TTX was also applied to several slices $(n=17)$, and in each case both the fiber volley and the EPSP components of both signals completely disappeared.

\section{Discussion}

\section{Selective cholinergic suppression of synaptic transmission in} CAI

The simulations of region CAl (Figs. 4-7) show that if the Schaffer collaterals store associations during cholinergic modulation, this requires greater cholinergic suppression of synaptic transmission in stratum radiatum (s. rad) than in stratum lacunosum-moleculare (s. 1-m). Brain slice experiments indicate that there is in fact a difference in the magnitude of cholinergic suppression of synaptic transmission between the two fiber lay- 
ers of region CA1. As illustrated in Figures $8-12$, there is a strong cholinergic suppression of synaptic transmission in $\mathbf{s}$. rad, and a comparatively weaker cholinergic suppression in s. $1-\mathrm{m}$. These results support the hypothesis that cholinergic modulation in region $\mathrm{CA} 1$ allows the Schaffer collaterals to store associations between activity in region CA3 and the afferent input from entorhinal cortex layer III. In addition, these results suggest that feedback regulation of cholinergic modulation may set the appropriate dynamics for learning or recall in cortical networks.

The effects of cholinergic modulation on synaptic transmission in stratum lacunosum-moleculare have not previously been characterized. The excitatory synaptic input to this region has not been the focus of much physiological research (see Colbert and Levy, 1992, for review), in contrast to the broad range of studies performed in stratum radiatum. However, previous physiological studies have demonstrated synaptic activation in region CAl with stimulation of the perforant path input to $\mathrm{s}$. 1-m (Andersen et al., 1966; Doller and Weight, 1982, 1985; Yeckel and Berger, 1990; Colbert and Levy, 1992a,b; Levy and Colbert, 1992), and anatomical studies have shown afferent input to stratum lacunosum-moleculare arising from layer III of the entorhinal cortex (Lorente de No, 1938; Steward, 1976; Witter et al., 1988). In the models presented here, this separate afferent input is essential if the Schaffer collaterals are to show associative memory function. As shown in the simulations, the s. 1-m input need not have a strong influence on CAl activity when studied in isolation. Input from the entorhinal cortex alone caused only moderate activity in CA1 neurons, but when combined with activity in region $\mathrm{CA} 3$, this input could effectively guide learning of associations by the Schaffer collaterals. In agreement with these results, anatomical evidence shows that the $s .1-m$ receives very little cholinergic innervation from the medial septum (Lynch et al., 1978; Green and Mesulam, 1988; Palacios and Mengod, 1989), suggesting that the effects of ACh on the synapses of s. $1-\mathrm{m}$ in vivo may not even be as strong as the suppression demonstrated in these experiments. A similar laminar selectivity has been demonstrated for the effects of baclofen in region CA1 (Colberl and Levy, 1992a).

The effects of cholinergic modulation in stratum radiatum are consistent with observations made in previous studies (Hounsgaard, 1978; Valentino and Dingledine, 1981; Rovira et al., 1983; Dutar and Nicoll, 1988; Sheridan and Sutor, 1990). Thus, considerable physiological evidence supports the strong cholinergic suppression of synaptic transmission in $\mathrm{s}$. rad that provides optimal heteroassociative memory function in the simulation. However, the dose-response plot presented in Figure 10 for the effects on synaptic transmission in s. rad differs somcwhat from that obtained by Sheridan and Sutor (1990). Their curve showed a $90 \%$ suppression at $10 \mu \mathrm{M}$ carbachol, a result obtained only for $100 \mu \mathrm{M}$ concentrations in this experiment. Other experiments on stratum radiatum did not display dose-response plots (Hounsgaard, 1978; Valentino and Dingledine, 1981; Rovira et al., 1983; Dutar and Nicoll, 1988), usually because ACh was applied iontophoretically to local regions of CA1, preventing accurate estimates of drug concentration (Hounsgaard, 1978; Valentino and Dingledine, 1981; Rovira et al., 1983). The ability of atropine, a specific muscarinic receptor antagonist, to block the cholinergic effects on synaptic transmission indicates that the suppression in both layers is mediated by muscarinic receptors. This specificity has previously been demonstrated in $\mathrm{s}$. rad (Valentino and Dingledine, 1981; Dutar and Nicoll, 1988; Sheridan and Sutor, 1990).

\section{Function of the Schaffer collaterals in hippocampal models}

The simulations and experimental results presented here support the hypothesis that the Schaffer collaterals store associations between activity in region CA3 and the afferent input from entorhinal cortex (Levy, 1989; Eichenbaum and Buckingham, 1990; Levy et al., 1990; McNaughton, 1991). While these results provide necessary support for the hypothesis, they do not rule out all other models of the function of region CA1. However, this evidence does run contrary to the hypothesis that the Schaffer collaterals exclusively undergo competitive self-organization (Rolls, 1991). The latter hypothesis neglects evidence for the direct perforant pathway projection from entorhinal cortex to region CA1. When this pathway is included in models, the change in influence mediated by cholinergic modulation suggests associative memory function of the Schaffer collaterals, though these synapses may undergo self-organization when cholinergic modulation is weak. The evidence presented here suggests that the input from entorhinal cortex to region CA1 is equal in importance to the Schaffer collaterals, and could be vital for understanding the significance of the broadly studied phenomenon of long-term potentiation in stratum radiatum (Gustafsson and Wigstrom, 1988). Further development of simulations such as this will be essential to linking the anatomical and physiological features of hippocampal function to more abstract theories proposed for the function of the hippocampus (Wickelgren, 1979; Levy, 1989; Levy et al., 1990; McClelland et al., 1992; Carpenter and Grossberg, 1993; Gluck and Myers, 1993). Convergence to a satisfactory description of the full range of data on hippocampal function will depend upon constraining models with anatomical and physiological data.

The selectivity of cholinergic suppression within region CA1 has particular significance in relation to physiological evidence that the input from entorhinal cortex to region CA1 is rather weak (Andersen et al., 1966; Colbert and Levy, 1992). In the experimental work presented here, s. $1-\mathrm{m}$ stimulation caused relatively small synaptic potentials, while s. rad stimulation easily evoked large EPSPs and population spikes. These results suggest that each of the fibers from region CA3 forms a much larger number of synapses on pyramidal cell dendrites than do the perforant path fibers in $\mathrm{s}$. $1-\mathrm{m}$. This connectivity difference might arise if synapses in $\mathrm{s}$. $1-\mathrm{m}$ undergo self-organization, with mechanisms minimizing the number of synaptic contacts. In contrast, associative memory function of synapses in s. rad would require a greater number of smaller synaptic influences. Greater cholinergic suppression of synaptic transmission in $s$. rad than s. 1-m might allow input from the entorhinal cortex to be the predominant influence on CAl activity during learning, allowing self-organization of the synapses of $s$. $1-m$ with heteroassociative memory function of the synapses of $\mathrm{s}$. rad. In addition, suppression of voltage- and calcium-dependent potassium currents in CA1 pyramidal cells will make these neurons more likely to fire action potentials in response to $\mathrm{s}$. 1-m input (Madison and Nicoll, 1984; Madison et al., 1987). Combined with the suppression in s. rad, this may increase the length constant of dendritic trees, further increasing the influence of the more distal s. 1-m synapses (Witter et al,, 1988).

Feedback regulation of cholinergic modulation sets appropriate dynamics for learning or recall

These are the first simulations to incorporate internal feedback regulation of cholinergic modulation. Most previous simula- 
tions of cortical memory function externally determine whether the network will respond to a stimulus as novel or as familiar. In particular, associative memory models set very different dynamics for learning of novel stimuli and recall of familiar stimuli (Anderson, 1983; Amit, 1988; Kohonen, 1988). Here this distinction between learning and recall can be regulated by the influence of cortical activity on the level of cholinergic modulation. For more familiar stimuli, the level of ACh decreases, and network activity depends less on external input and more on the recall of previous associations. For novel stimuli, the level of ACh increases, and network activity depends more on external input than recall activity, setting appropriate dynamics for learning. This simulation suggests a continuum between learning and recall, with potential intermediate states in which partial recall could speed new learning. The feedback regulation of the dynamics of learning and recall depends upon the combination of a number of different neuromodulatory influences of ACh. These simulations are the first to incorporate influences of cholinergic modulation on inhibitory synaptic transmission (Pitler and Alger, 1992) and on the rate of synaptic modification (Blitzer et al., 1990; Barkai et al., 1993; Huerta and Lisman, 1993). Previous simulations have explored the effect of cholinergic modulation of synaptic transmission (Hasselmo et al., 1992; present results) and neuronal adaptation (Barkai and Hasselmo, 1994a; Hasselmo et al., 1993).

The selective cholinergic suppression of synaptic transmission was previously demonstrated in the piriform cortex (Hasselmo and Bower, 1992) and its significance for autoassociative memory function has been extensively studied in models of the piriform cortex, using analytical representations (Hasselmo, 1993, 1994), biophysical compartmental simulations (Barkai and Hasselmo, 1994a,b; Hasselmo et al., 1993), and more abstract computational models (Hasselmo et al., 1992; Hasselmo, 1993, 1994). In addition, the cholinergic suppression of neuronal adaptation has been demonstrated in the piriform cortex (Tseng and Haberly, 1988; Barkai and Hasselmo, 1994a) and has been shown to enhance associative memory function in biophysical compartmental simulations (Barkai and Hasselmo, 1994b; Hasselmo et al., 1993). Cholinergic modulation in the piriform cortex may make afferent fibers from the olfactory bulb the predominant influence during learning in piriform cortex, allowing these fibers to undergo self-organization while intrinsic fibers have associative memory function (Hasselmo, 1994). The results presented here, along with previous analytical work (Hasselmo, 1994), show that these results apply to heteroassociative memory function as well.

Insufficient cholinergic suppression of synaptic transmission during learning can allow runaway synaptic modification of excitatory synapses (Hasselmo et al., 1992; Barkai and Hasselmo, 1994b; Hasselmo, 1993, 1994). This phenomenon has been suggested to underlie the progression of neuronal degeneration in cortical structures in Alzheimer's disease (Hasselmo, 1994). Runaway synaptic modification can occur at the $s$. rad synapses in the simulation presented here. However, the pyramidal cells of region CA3 show relatively less formation of neurofibrillary tangles in Alzheimer's disease, whereas pyramidal cells of region CAl near the border of the subiculum show early appearance and high density of neurofibrillary tangles (Hyman et al., 1987). This suggests that the greater sensitivity of CA1 pyramidal cells to development of neurofibrillary tangles may be due to postsynaptic influences of runaway synaptic modification (e.g., excitotoxicity), or that the runaway synaptic modification may chiefly occur on the synapses projecting from region CA1 back to the subiculum and entorhinal cortex. Interestingly, in some cases of Alzheimer's disease, increased levels of cholinergic marker enzymes appear in s. 1-m of region CA1 and the subiculum (Hyman et al., 1987). This suggests that a possible compensatory mechanism to reduce runaway synaptic modification in Alzheimer's disease might be to increase the cholinergic suppression in s. 1-m. As shown in Figure $5 B$, this will slow the rate of synaptic modification in region $\mathrm{CA} 1$.

The results presented here are relevant to a more abstract model of cortical function termed Adaptive Resonance Theory, or ART (Carpenter and Grossberg, 1993). An important feature of those models concerns the solution of the stability/plasticity dilemma. To avoid instability in the formation of categories, a component of ART networks termed the orienting subsystem compares input with previously learned categories. If the match does not exceed a criterion level, a new category is formed. The architecture and implementation of these networks are considerably different from the simulations presented here of region $\mathrm{CA} 1$, but the computational issues are similar. The orienting subsystem has been presented previously as a theoretical description of the role of the hippocampus in cortical function (Carpenter and Grossberg, 1993).

\section{Anatomy and physiology of the feedback regulation of cholinergic modulation}

The simulation presented here describes a possible function for the feedback regulation of cholinergic modulation in the hippocampus. The cholinergic modulation of region $\mathrm{CAl}$ arises from the medial septum and the vertical limb of the diagonal band of Broca (Frotscher and Leranth, 1985). For many years, this modulation was considered to be under inhibitory control from the lateral septum, since circumstantial anatomical evidence suggested that cholinergic neurons of the medial septum received GABAergic input from the lateral septum (Leranth and Frotscher, 1989). This would allow increased hippocampal activity to downregulate cholinergic modulation, since CA1 and CA3 pyramidal cells project fibers to the lateral septum (Swanson and Cowan, 1979), forming asymmetric (presumably excitatory) connections onto the cells located there (Leranth and Frotscher, 1989). However, recent evidence suggests that the medial septum may not be under direct inhibitory control by the lateral septum (Leranth et al., 1992). Instead, the medial septum appears to receive direct inhibitory projections from regions CA3 and CAl of the hippocampus (Toth et al., 1993).

Physiological studies have demonstrated that stimulation of efferent fibers of the hippocampus in the fimbria inhibits the activity of neurons in the medial septum (McLennan and Miller, 1974a,b). Significantly, Givens and Olton (1990) have found that GABAergic inhibition in the medial septum, evoked by in vivo infusions of the $\mathrm{GABA}_{\mathrm{A}}$ agonist muscimol, impaired working memory in ways similar to those caused by blocking cholinergic modulation in the hippocampus. Inactivation of the Inedial septum with lidocaine has been shown to impair working memory without significantly changing spatial selectivity or mean discharge rate of region CA1 neurons (Mizumori et al., 1989), suggesting that this manipulation may set the dynamics of CA1 activity to constant recall. With medial septum inactivation, removal of the cholinergic suppression of synaptic transmission in s. rad allows completion of sparse CA3 activity to drive CA 1 neurons, but may lock the network into a state of recall, preventing new information from being incorporated. 
Further experiments are necessary to test this model of the feedback regulation of cholinergic modulation. The model predicts that cholinergic neurons of the medial septum should be selectively active during presentation of novel stimuli. Recordings from neurons of the diagonal band in monkeys during serial presentation of objects have demonstrated neurons in this region with selective responses to novel but not familiar stimuli (Wilson and Rolls, 1990). Further experiments are necessary to verify that medial septal activity will start high and decrease during learning. In addition, experiments should test the hypothesis that medial septal cholinergic output will be lowest when CAl contains strong but relatively sparse activity.

\section{Cholinergic modulation in other cortical areas}

ACh may have similar modulatory effects in all cortical regions. In addition to region $\mathrm{CAl}$ and the piriform cortex, cholinergic suppression of synaptic transmission has been demonstrated in other hippocampal regions, including the dentate gyrus (Yamamoto and Kawai, 1967; Kahle and Cotman, 1989) and region CA3 (Valentino and Dingledine, 1981; Williams and Johnston, 1990). In the dentate gyrus, Kahle and Cotman (1989) demonstrated a $50 \%$ suppression of synaptic transmission in the middle molecular layer at $100 \mu \mathrm{M}$ carbachol, with no suppression in the outer molecular layer. The simulations presented here suggest that this selective cholinergic modulation in the dentate gyrus would allow input to the middle molecular layer from the medial entorhinal cortex to have associative memory function, while allowing the lateral entorhinal cortex input to the outer molecular layer to undergo self-organization. It is interesting to note that in these cases of selectivity of cholinergic suppression, the input to the proximal portion of pyramidal cell dendrites has been more strongly suppressed (Kahle and Cotman, 1989; Hasselmo and Bower, 1992; present results). This suggests that the loss of proximal synaptic currents, and the associated increase in dendritic length constant, may be an important factor in the functional characteristics of these regions.

The innervation of the hippocampal formation from the entorhinal cortex shows a characteristic topography, with fibers from the lateral entorhinal cortex terminating in the portion of CA1 adjacent to the subiculum, and fibers from the medial entorhinal cortex terminating in the portion of CAl adjacent to CA3 (Steward, 1976; Steward and Scoville, 1976; Witter et al., 1988). In the dentate gyrus, fibers from the lateral and medial entorhinal cortex show different pharmacological sensitivity to ACh (Kahle and Cotman, 1989), L-AP4 (Koerner and Cotman, 1981), and baclofen (Lanthorn and Cotman, 1990), suggesting that the perforant path innervation of different portions of $s$. $1-m$ may differ physiologically as well. The experiments presented here involved recording between one-third and one-half of the distance from the subiculum to the border of CA3. However, preliminary experiments did not demonstrate any differences in the sensitivity of synaptic potentials recorded near the border of the subiculum in comparison to potentials recorded near the border of CA3. This lack of a difference is not inconsistent with the results of Kahle and Cotman, since the fibers innervating $\mathrm{s}$. $1-\mathrm{m}$ arise from pyramidal cells in layer III of entorhinal cortex (Steward and Scoville, 1976), rather than stellate neurons in layer II.

Cholinergic suppression of synaptic transmission should also be of considerable importance for the function of region CA3, which has been proposed to have an autoassociative-type function dependent upon modification of the longitudinal associa- tion pathway synapsing in stratum radiatum (Marr, 1971; McNaughton and Morris, 1987; Levy, 1989; McNaughton, 1991; Treves and Rolls, 1991). Cholinergic suppression of synaptic transmission has been reported in region CA3 (Valentino and Dingledine, 1981), but the laminar selectivity of this suppression has not been explored. If region CA3 performs autoassociative storage of patterns determined by input from other regions, this would require weaker suppression of synaptic transmission at the mossy fibers in stratum lucidum and at the perforant path synapses in stratum lacunosum-moleculare, compared to stronger suppression at synapses in stratum radiatum. In addition, region CA3 should have mechanisms for feedback regulation of cholinergic modulation from the medial septum. Suppression of synaptic transmission at the mossy fibers has been reported (Williams and Johnston, 1990), but appears to be weaker than the suppression reported in stratum radiatum (Valentino and Dingledine, 1981). However, a direct comparison of the level of suppression between stratum lucidum, stratum radiatum, and stratum lacunosum-moleculare in region CA3 has not been presented.

\section{References}

Alger BE, Dhanjal SS, Dingledine R, Garthwaite J, Henderson G, King GL, Lipton P, North A, Schwartzkroin PA, Sears TA, Segal M, Wittingham TS, Williams J (1984) Brain slice methods. In: Brain slices (Dingledine R, ed), pp 381-437. New York: Plenum.

Amaral DG, Witter MP (1989) The 3-dimensional organization of the hippocampal formation - a review of anatomical data. Neuroscience 31:571-591.

Amit DJ (1988) Modeling brain function: the world of attractor neural networks. Cambridge: Cambridge UP.

Andersen P, Holmqvist B, Voorhoeve PE (1966) Excitatory synapses on hippocampal apical dendrites activated by entorhinal stimulation. Acta Physiol Scand 66:461-472.

Anderson JA (1972) A simple neural network generating an interactive memory. Math Biosci 14:197-220.

Anderson JA (1983) Cognitive and psychological computation with neural models. IEEE Trans SMC-13:799-815.

Barkai E, Hasselmo ME (1994a) Modulation of the input/output function of rat piriform cortex pyramidal cells. J Neurophysiol, in press.

Barkai E, Hasselmo ME (1994b) Modulation of associative memory function in a biophysical simulation of rat piriform cortex. J Neurophysiol, in press.

Barkai E, Horwitz G, Bergman RE, Hasselmo ME (1993) Long-term potentiation and associative memory function in a biophysical simulation of piriform cortex. Soc Neurosci Abstr 19:376.3.

Beatty WW, Carbone CP (1980) Septal lesions, intramaze cues and spatial behavior in rats. Physiol Behav 24:675-678.

Benardo LS, Prince DA (1982) Ionic mechanisms of cholinergic excitation in mammalian hippocampal pyramidal cells. Brain Res 249: 333-344.

Bergman RE, Vanier ME, Horwitz G, Bower JM, Hasselmo ME (1993) Cholinergic modulation of associative memory function in a realistic computational model of piriform cortex. In: Computation and neural systems (Eeckman F, Bower JM, eds), pp 273-280. Norwell, MA: Kluwer.

Bland BH, Colom LV (1993) Extrinsic and intrinsic properties underlying oscillation and synchrony in limbic cortex. Prog Neurobiol 41:157-208.

Blitzer RD, Gil O, Landau EM (1990) Cholinergic stimulation enhances long-term potentiation in the $\mathrm{CA} 1$ region of rat hippocampus. Neurosci Lett 119:207-210.

Brocher S, Artola A, Singer W (1992) Agonists of cholinergic and noradrenergic receptors facilitate synergistically the induction of longterm potentiation in slices of rat visual cortex. Brain Res 573:27-36.

Burgard EC, Sarvey JM (1990) Muscarinic receptor activation facilitates the induction of long-term potentiation (LTP) in the rat dentate gyrus. Neurosci Lett 116:34-39.

Carpenter GA, Grossberg S (1993) Normal and amnesic learning, recognition and memory by a neural model of cortico-hippocampal interactions. Trends Neurosci 16:131-137. 
Colbert CM, Levy WB (1992a) Electrophysiological and pharmacological characterization of perforant path synapses in CAl: mediation by glutamate receptors. J Neurophysiol $68: 1-8$.

Colbert CM, Levy WB (1992b) GABA-A inhibition opposes monosynaptic perforant-path excitation of CA1 pyramids. Soc Neurosci Abstr 18:628.13.

Doller HJ, Weight FF (1982) Perforant pathway activation of hippocampal CAl stratum pyramidale neurons: electrophysiological evidence for a direct pathway. Brain Res 237:1-13.

Doller HJ, Weight FF (1985) Perforant pathway-evoked long-term potentiation of CAl neurons in the hippocampal slice preparation. Brain Res 333:305-310.

Dutar P, Nicoll RA (1988) Classification of muscarinic responses in hippocampus in terms of receptor subtypes and 2nd-messenger systems-clcctrophysiological studies in vitro. J Neurosci 8:4214 4224.

Eichenbaum H, Buckingham J (1990) Studies on hippocampal processing: experiment, theory and model. In: Learning and computational neuroscience: foundations of adaptive networks (Gabriel $M$, Moore J, eds), pp 171-231. Cambridge, MA: MIT Press.

Frotscher M, Leranth C (1985) Cholinergic innervation of the rat hippocampus as revealed by choline acetyltransferase immunocytochemistry: a combined light and electron microscopic study. J Comp Neurol 239:237-246.

Gaykema RPA, Luiten PGM, Nyakas C, Traber J (1990) Cortical projection patterns of the medial septum-diagonal band complex. J Comp Neurol 293:103-124.

Givens BS, Olton DS (1990) Cholinergic and GABAergic modulation of the medial septal area: effect on working memory. Behav Neurosci 104:849-855.

Gluck MA, Myers CE (1993) Hippocampal mediation of stimulus representation: a computational theory. Hippocampus 3:491-516.

Green RC, Mesulam MM (1988) Acetylcholinesterase fiber staining in the human hippocampus and parahippocampal gyrus. J Comp Neurol 273:488-499.

Grossberg S (1970) Some networks that can learn, remember and reproduce any number of complicated space-time patterns, II. Stud Appl Math 49:135-166.

Grossberg S (1976) On the development of feature detectors in the visual cortex with applications to learning and reaction-diffusion systems. Biol Cybern 21:145-159.

Gustafsson B, Wigstrom H (1988) Physiological mechanisms underlying long-term potentiation. Trends Neurosci 11:156-162.

Hagan JJ, Morris RGM (1989) The cholinergic hypothesis of memory: a review of animal experiments. In: Psychopharmacology of the aging nervous system (Iversen LL, Iversen SD, Snyder SH, eds), pp 237324. New York: Plenum.

Hasselmo ME (1993) Acetylcholine and learning in a cortical associative memory. Neural Comput 5:32-44.

Hasselmo ME (1994) Runaway synaptic modification in models of cortex: implications for Alzheimer's disease. Neural Networks 7:1340.

Hasselmo ME, Bower JM (1991) Selective suppression of afferent but not intrinsic fiber synaptic transmission by 2-amino-4-phosphonobutyric acid (AP4) in piriform cortex. Brain Res 548:248-255.

Hasselmo ME, Bower JM (1992) Cholinergic suppression specific to intrinsic not afferent fiber synapses in rat piriform (olfactory) cortex. J Neurophysiol 67:1222-1229.

Hasselmo ME, Bower JM (1993) Acetylcholine and memory. Trends Neurosci 16:218-222.

Hasselmo ME, Anderson BP, Bower JM (1992) Cholinergic modulation of cortical associative memory function. J Neurophysiol 67: 1230-1246.

Hasselmo ME, Barkai E, Horwitz G, Bergman RE (1993) Modulation of neuronal adaptation and cortical associative memory function. In: Computation and neural systems II (Eeckman F, Bower JM, cds), in press. Norwell, MA: Kluwer.

Hounsgaard J (1978) Presynaptic inhibitory action of acetylcholine in area CA1 of the hippocampus. Exp Neurol 62:787-797.

Huerta PT, Lisman JE (1993) Heightened synaptic plasticity of hippocampal CAl neurons during a cholinergically induced rhythmic state. Nature 364:723-725.

Hyman BT, Kromer LJ, Van Hoesen GW (1987) Reinnervation of the hippocampal perforant pathway zone in Alzheimer's disease. Ann Neurol 21:250-267.

Kahle JS, Cotman CW (1989) Carbachol depresses the synaptic re- sponses in the medial but not the lateral perforant path. Brain Res 482:159-163.

Kelso SR, Ganong AH, Brown TH (1986) Hebbian synapses in the hippocampus. Proc Natl Acad Sci USA 83:5326-5330.

Koerner JF, Cotman CW (1981) Micromolar L-2-amino-4-phosphonobutyric acid selectively inhibits perforant path synapses from lateral entorhinal cortex. Brain Res 216:192-198.

Kohonen T (1972) Correlation matrix memories. IEEE Trans C-21: 353-359.

Kohonen T (1988) Self-organization and associative memory. Berlin: Springer

Kopelman MD (1986) The cholinergic neurotransmitter system in human memory and dementia: a review. Q J Exp Psychol 38:535573.

Kunkel DD, Lacaille JC, Schwartzkroin PA (1988) Ultrastructure of stratum lacunosum-moleculare interneurons of hippocampal CA1 region. Synapse 2:382-394.

Lacaille JC, Schwartzkroin PA (1988) Stratum lacunosum-moleculare interneurons of hippocampal CAl region. I. Intracellular response characteristics, synaptic responses and morphology. J Neurosci 8:14001410 .

Lanthorn TH, Cotman CW (1981) Baclofen selectively inhibits excitatory synaptic transmission in the hippocampus. Brain Res 225: 171-178.

Leranth C, Frotscher M (1989) Organization of the septal region in the rat brain: cholinergic-GABAergic interconnections and the termination of hippocampo-septal fibers. J Comp Neurol 289:304-314.

Leranth C, Deller T, Buzsaki G (1992) Intraseptal connections redefined-lack of lateral septum to medial septum path. Brain Res 583: $1-11$.

Levy WB (1989) A computational approach to hippocampal function. In: Computational models of learning in simple neural systems (Ilawkins RD, Bower GH, eds), pp 243-305. Orlando, FL: Academic.

Levy WB, Colbert CM (1992) Associative potentiation of Schaffer collaterals by paired conditioning of the perforant path in hippocampal CA1. Soc Neurosci Abstr 18:628.14.

Levy WB, Colbert CM, Desmond NL (1990) Elemental adaptive processes of neurons and synapses: a statistical/computational perspective. In: Neuroscience and connectionist theory (Gluck MA, Rumelhart DE, eds), pp 187-236. Hillsdale, N.: Erblaum.

Liljenstrom H, Hasselmo ME (1993) Acetylcholine and cortical oscillatory dynamics. In: Computation and neural systems (Eeckman $\Gamma$, Bower JM, eds), pp 523-530. Norwcll, MA: Kluwer.

Linsker R (1988) Self-organization in a perceptual network. Computer 21:105-117.

Lorente de No R (1934) Studies on the structure of the cerebral cortex. II. Continuation of the study of the ammonic system. J Psychol Neurol 46:113-177.

Lynch G, Rose G, Gall C (1978) Anatomical and functional aspects of the septo-hippocampal projections. In: Functions of the septohippocampal system. Amsterdam: Elsevier.

Madison DV, Nicoll RA (1984) Control of the repetitive discharge of rat CA1 pyramidal neurones in vitro. J Physiol (Lond) 354:319-331.

Madison DV, Lancaster B, Nicoll RA (1987) Voltage clamp analysis of cholinergic action in the hippocampus. J Neurosci 7:733-741.

Markram J, Segal M (1990) Acetylcholine potentiates responses to $N$-methyl-D-aspartate in the rat hippocampus. Neurosci Lett 1 13:6265.

Marr D (1971) Simple memory: a theory for archicortex. Philos Trans R Soc Lond [Biol] B262:23-81.

McClelland JL, McNaughton BL, O'Reilly R, Nadel L (1992) Complementary roles of hippocampus and neocortex in learning and memory. Soc Neurosci Abstr 18:508.7.

McLennan H, Miller JJ (1974a) The hippocampal control of neuronal discharges in the septum of the rat. J Physiol (Lond) 237:607-624.

McLennan H, Miller JJ (1974b) Gamma-aminobutyric acid and inhibition in the septal nuclei of the rat. J Physiol (Lond) 237:625-633.

McNaughton BL (1991) Associative pattem completion in hippocampal circuits: new evidence and new questions. Brain Res Rev 16:193220.

McNaughton BL, Morris RGM (1987) Hippocampal synaptic enhancement and information storage within a distributed memory system. Trends Neurosci 10:408-415.

Miller KD, Keller JB, Stryker MP (1989) Ocular dominance column development-analysis and simulation. Science 245:605-615. 
Mizumori SJY, McNaughton BL, Barnes CA, Fox KB (1989) Preserved spatial coding in hippocampal CA1 pyramidal cells during reversible suppression of $\mathrm{CA} 3 \mathrm{c}$ output: evidence for pattern completion in hippocampus. J Neurosci 9:3915-3928.

Palacios JM, Mengod G (1989) Radiohistochemistry of receptors in the hippocampus: focus on the cholinergic receptors. In: The hippocampus: new vistas (Chan-Palay V, Kohler C, eds), pp 207-224. New York: Liss.

Pitler TA, Alger BE (1992) Cholinergic excitation of GABAergic interneurons in the rat hippocampal slice. J Physiol (Lond) 450:127142.

Rolls ET (1991) The representation and storage of information in neuronal networks in the primate cerebral cortex and hippocampus. In: The computing neuron (Durbin R, Miall C, Mitchison $\mathrm{G}$, eds), pp 125-159. Wokingham, UK: Addison-Wesley.

Rovira C, Ben-Ari Y, Cherubini E, Krnjevic K, Ropert N (1983) Pharmacology of the dendritic action of acetylcholine and further observations on the somatic disinhibition in the rat hippocampus in situ. Neuroscience 8:97-106.

Sheridan RD, Sutor B (1990) Presynaptic M1 muscarinic cholinoceptors mediate inhibition of excitatory synaptic transmission in the hippocampus in vitro. Neurosci Lett 108:273-278.

Spencer DG, Pontecorvo MJ, Heise GA (1985) Central cholinergic involvement in working memory: effects of scopolamine on continuous nonmatching and discrimination performance in the rat. Behav Neurosci 99:1049-1065.

Steward O (1976) Topographic organization of the projections from the entorhinal areas of the hippocampal formation of the rat. J Comp Neurol 167:285-314.

Steward O, Scoville SA (1976) Cells of origin of entorhinal cortical afferents to the hippocampus and fascia dentate of the rat. J Comp Ncurol 169:347-370.

Swanson LW, Cowan WM (1979) Connections of the septal region in the rat. J Comp Neurol 186:621-656.

Toth K, Borhegyi Z, Freund TF (1993) Postsynaptic targets of GABAergic hippocampal neurons in the medial septum diagonal band of Broca complex. J Neurosci 13:3712-3724.

Traub R, Miles R, Buzsaki G (1992) Computer simulation of carbachol-driven rhythmic population oscillations in the CA3 region of the in vitro rat hippocampus. J Physiol (Lond) 451:653-672.

Treves A, Rolls ET (1991) What determines the capacity of autoassociative memories in the brain? Network 2:371-397.
Umbriaco D, Watkins KC, Descarries L, Cozzari C, Hartman BK (1992) Serial section analysis of the acetylcholine $(\mathrm{ACh})$ innervation in adult rat parietal cortex. Soc Neurosci Abstr 18:976.

Valentino RJ, Dingledine R (1981) Presynaptic inhibitory effect of acetylcholine in the hippocampus. J Neurosci 1:784-792.

von der Malsburg C (1973) Self-organization of orientation sensitive cells in the striate cortex. Kybernetik 14:85-100.

Walker JA, Olton DS (1984) Fimbria-fornix lesions impair spatial working memory but not cognitive mapping. Behav Neurosci 98:226242.

Wickelgren WA (1979) Chunking and consolidation: a theoretical synthesis of semantic networks, configuring in conditioning, S-R versus cognitive learning, normal forgetting, the amnesic syndrome and the hippocampal arousal system. Psychol Rev 86:44-60.

Wigstrom H, Gustafsson B, Huang Y-Y, Abraham WC (1986) Hippocampal long-term potentiation is induced by pairing single afferent volleys with intracellularly injected depolarizing current pulses. Acta Physiol Scand 126:317-319.

Williams SH, Constanti A (1988) Quantitative effects of some muscarinic agonists on evoked surface-negative field potentials recorded from the guinea-pig olfactory cortex slice. $\mathrm{Br} \mathrm{J}$ Pharmacol 93:846854.

Williams SH, Johnston D (1990) Muscarinic depression of synaptic transmission at the hippocampal mossy fiber synapse. J Neurophysiol 64:1089-1097.

Wilson FAW, Rolls ET (1990) I earning and memory is reflected in the responses of reinforcement-related neurons in the primate basal forebrain. J Neurosci 10:1254-1267.

Wilson MA, Bower JM (1992) Cortical oscillations and temporal interactions in a computer simulation of piriform cortex. J Neurophysiol 67:981-995.

Witter MP, Griffioen AW, Jorritsma-Byham B, Krijnen JLM (1988) Entorhinal projections to the hippocampal CAI region in the rat: an underestimated pathway. Neurosci Lett 85:193-198.

Yamamoto C, Kawai N (1967) Presynaptic action of acetylcholine in thin sections from the guinea-pig dentate gyrus in vitro. Exp Neurol 19:176-187.

Yeckel MF, Berger TW (1990) Feedforward excitation of the hippocampus by afferents from the entorhinal cortex: redefinition of the role of the trisynaptic pathway. Proc Natl Acad Sci IJSA 87:58325836 . 